\title{
On the structure of Langmuir turbulence
}

Article

Accepted Version

Teixeira, M.A.C. and Belcher, S. E. (2010) On the structure of Langmuir turbulence. Ocean modelling, 31 (3-4). pp. 105-119. ISSN 1463-5003 doi:

https://doi.org/10.1016/j.ocemod.2009.10.007 Available at https://centaur.reading.ac.uk/16602/

It is advisable to refer to the publisher's version if you intend to cite from the work. See Guidance on citing.

To link to this article DOI: http://dx.doi.org/10.1016/j.ocemod.2009.10.007

Publisher: Elsevier

All outputs in CentAUR are protected by Intellectual Property Rights law, including copyright law. Copyright and IPR is retained by the creators or other copyright holders. Terms and conditions for use of this material are defined in the End User Agreement.

\section{www.reading.ac.uk/centaur}

\section{CentAUR}

Central Archive at the University of Reading

Reading's research outputs online 


\title{
On the structure of Langmuir turbulence
}

\author{
M. A. C. Teixeira ${ }^{\mathrm{a}, *}$, S. E. Belcher ${ }^{\mathrm{b}}$ \\ ${ }^{a}$ University of Lisbon, CGUL, IDL, Lisbon, Portugal \\ ${ }^{b}$ Department of Meteorology, University of Reading, Reading, UK
}

\begin{abstract}
The Stokes drift induced by surface waves distorts turbulence in the winddriven mixed layer of the ocean, leading to the development of streamwise vortices, or Langmuir circulations, on a wide range of scales. We investigate the structure of the resulting Langmuir turbulence, and contrast it with the structure of shear turbulence, using rapid distortion theory (RDT) and kinematic simulation of turbulence. Firstly, these linear models show clearly why elongated streamwise vortices are produced in Langmuir turbulence, when Stokes drift tilts and stretches vertical vorticity into horizontal vorticity, whereas elongated streaky structures in streamwise velocity fluctuations $(u)$ are produced in shear turbulence, because there is a cancellation in the streamwise vorticity equation and instead it is vertical vorticity that is amplified. Secondly, we develop scaling arguments, illustrated by analysing data from LES, that indicate that Langmuir turbulence is generated when the deformation of the turbulence by mean shear is much weaker than the deformation by the Stokes drift. These scalings motivate a quantitative RDT
\end{abstract}

\footnotetext{
*Corresponding author: M. A. C. Teixeira, Centro de Geofísica da Universidade de Lisboa, Edifício C8, Campo Grande, 1749-016 Lisbon, Portugal

Email addresses: mateixeira@fc.ul.pt (M. A. C. Teixeira)
} 
model of Langmuir turbulence that accounts for deformation of turbulence by Stokes drift and blocking by the air-sea interface that is shown to yield profiles of the velocity variances $\left(\overline{u^{2}}, \overline{v^{2}}, \overline{w^{2}}\right)$ in good agreement with LES. The physical picture that emerges, at least in the LES, is as follows. Early in the life cycle of a Langmuir eddy initial turbulent disturbances of vertical vorticity are amplified algebraically by the Stokes drift into elongated streamwise vortices, the Langmuir eddies. The turbulence is thus in a near two-component state, with $\overline{u^{2}}$ suppressed and $\overline{v^{2}} \approx \overline{w^{2}}$. Near the surface, over a depth of order the integral length scale of the turbulence, the vertical velocity $(w)$ is brought to zero by blocking of the air-sea interface. Since the turbulence is nearly two-component, this vertical energy is transfered into the spanwise fluctuations, considerably enhancing $\overline{v^{2}}$ at the interface. After a time of order half the eddy decorrelation time the nonlinear processes, such as distortion by the strain field of the surrounding eddies, arrest the deformation and the Langmuir eddy decays. Presumably, Langmuir turbulence then consists of a statistically steady state of such Langmuir eddies. The analysis then provides a dynamical connection between the flow structures in LES of Langmuir turbulence and the dominant balance between Stokes production and dissipation in the turbulent kinetic energy budget, found by previous authors.

Key words: Langmuir circulation, Turbulence, Stokes drift, Mixed layer, Rapid distortion theory 


\section{Introduction}

The velocity field in the surface mixed layer of the ocean is often dominated by longitudinal vortices, known as Langmuir circulations, which are aligned roughly with the wind, as reviewed by Leibovich (1983), Garrett (1996) and Thorpe (2004). Direct observations (Pluddemann et al., 1996;

Smith, 1998; D'Asaro, 2001) and laboratory experiments (Faller and Cartwright, 1983; Nepf and Monismith, 1991; Melville et al., 1998) have shown that these circulations play a key role in the transport and mixing of momentum, heat, pollutants and dissolved gases from the surface into the deeper ocean (Kantha and Clayson, 2004; McWilliams and Sullivan, 2001), and also in the dispersion of buoyant tracers trapped at the surface (Faller and Auer, 1988; Thorpe, 2001).

Craik and Leibovich (1976) and later Craik (1977), Leibovich (1977, 1980), Leibovich and Radhakrishnan (1977) and Leibovich and Paolucci (1981) developed the established model for growth of Langmuir circulations from small flow perturbations, namely an instability of the wind-induced shear current to the Stokes drift associated with the surface waves. According to this mechanism (known as CL2) the Stokes drift tilts and amplifies the vertical vorticity associated with any small spanwise variations of the shear current. This amplified vorticity then further amplifies the perturbation to the wind-induced shear flow, leading to instability. The CraikLeibovich analysis, and the body of work that has grown from it (see Leibovich, 1983; Thorpe, 2004), is concerned with the initiation of Langmuir circulations within a flow where any existing turbulence has a purely diffusive effect, and yields the spatial structure of the unstable normal modes and 
the scale and growth rate of the most unstable mode.

In the ocean mixed layer the flow is usually fully turbulent, and the Langmuir circulations are best viewed as coherent structures embedded within the turbulence: the observations of Faller and Auer (1988), Pluddemann et al. (1996) and Smith (1998) speak of Langmuir circulations with inherent randomness and on a whole spectrum of scales. Large eddy simulation (LES) studies have lent support to this picture. Skillingstad and Denbo (1995), McWilliams et al. (1997) (henceforth MSM97), and later McWilliams and Sullivan (2001) and Skyllingstad (2001), have simulated Langmuir turbulence using LES of the full Navier-Stokes equations, with an eddy viscosity to represent the energy lost at small scales. Following Craik and Leibovich, in all these studies the effects of the surface waves were represented through the deformation of the vorticity by the Stokes drift - via the so-called vortex force. In this sense these simulations are LES of the Craik-Leibovich equations. Noh and Min (2004) additionally included a simple representation of wave breaking, and Li et al. (2005) carefully investigated the flow regimes between three limit situations, where the flow is dominated by shear, surface waves, or convection.

Recent analysis of LES of Langmuir turbulence by Polton and Belcher (2007) has shown that within the Stokes layer, where the Stokes drift acts, the dominant balance in the turbulent kinetic energy (TKE) budget is between Stokes production of TKE and dissipation (at least when wave breaking is absent or unimportant). Deeper into the layer the budget is dominated by turbulent transport of TKE from the Stokes layer and dissipation, which Polton and Belcher (2007) attribute to downwelling jets associated with con- 
vergence zones between vortices in the Stokes layer. Grant and Belcher (2009) further analysed the TKE budget and suggested that it scales on the velocity $w_{* L}=\left(u_{*}^{2} U_{S 0}\right)^{\frac{1}{3}}$ (where $u_{*}$ and $U_{S 0}$ are, respectively, the friction and surface Stokes drift velocities), and the length $h$, the depth of the mixed layer although many alternative scalings have been proposed (Pluddemann et al., 1996; Smith, 1998; D'Asaro, 2001; Harcourt and D'Asaro, 2008). Grant and Belcher (2009) then showed that, when scaled by these variables, the profiles of dissipation rate, velocity variances and other turbulence quantities collapse to single profiles for a variety of forcing variables. This scaling demonstrates that Langmuir turbulence is an asymptotically distinct state of turbulence that has fundamentally different dynamics to a shear driven layer. At this same time some authors (e.g. Tsai et al., 2005) have computed LES of a stress driven layer, without wave forcing, and argued that some of the features usually attributed to Langmuir turbulence are also present in shear turbulence.

The first aim of the present paper is therefore to analyse the processes that shape turbulence distorted by Stokes drift and the contrast with processes that shape turbulence in a shear flow. A linear rapid distortion theory (hereafter RDT) model is used to illustrate these changes.

There are a range of processes acting even within the simplified system computed by the LES studies: mean shear generates turbulence, the Stokes drift deforms turbulence, and the air-water interface blocks the turbulence. So the second aim of this paper is to establish the dynamical processes responsible for shaping Langmuir turbulence. This is done by building upon the RDT model for the distortion of turbulence by a travelling surface wave 
developed by Teixeira and Belcher (2002) (henceforth TB2002), and making quantitative comparisons with the results of LES. We will find that the linearised rapid-distortion approach explains both qualitative and quantitative features of Langmuir turbulence. In this sense we seek here to provide a dynamical connection between the results from the TKE budget and the flow structures observed in Langmuir turbulence.

The remainder of the paper is organised as follows. In Section 2, we begin by introducing the formulation of the RDT model and contrasting the flow structure predicted by kinematic simulation of turbulence (KST) for shear turbulence and turbulence distorted by a surface wave. In Section 3 we develop scalings for Langmuir turbulence through examination of the results from the LES of MSM97. These scalings motivate a specific quantitative comparison with RDT and KST. The ensuing results are presented in Section 4. Finally, the main conclusions of this study are presented in Section 5.

\section{Rapid distortion and kinematic simulation of Langmuir turbu- lence}

In RDT, the equations of motion are linearised with respect to the turbulent quantities (Batchelor and Proudman, 1954). Some turbulence is assumed to exist initially, which is distorted for a finite time by an external forcing (e.g. the Stokes drift of a wave) according to the linear dynamics. A final turbulence state is thus obtained. This approach has been used previously for shear flows by Townsend (1970), Lee and Hunt (1989), Lee et al. (1990) and Mann (1994), and for blocking by rigid boundaries by Hunt and Graham (1978) and Magnaudet (2003), for example. The limitations and assumptions 
underlying RDT have been reviewed by Batchelor and Proudman (1954) and Hunt (1973). Essentially, since RDT neglects nonlinear interactions in the turbulence, it is approximately valid in situations when the velocity scale and strain rate of the mean distorting flow are considerably larger than those of the turbulence (i.e. weak turbulence).

For an incompressible and non-rotating fluid at high Reynolds number, the linearised momentum and mass conservation equations may then be written

$$
\begin{aligned}
& \frac{\partial \mathbf{u}}{\partial t}+\mathbf{U} \cdot \nabla \mathbf{u}+\mathbf{u} \cdot \nabla \mathbf{U}=\frac{1}{\rho} \nabla p, \\
& \nabla \cdot \mathbf{u}=0
\end{aligned}
$$

where $\mathbf{u}$ is the turbulent velocity, $p$ is the turbulent pressure, $\mathbf{U}$ is the mean velocity, $\rho$ is the density and $\nabla$ is the spatial gradient operator. Mixing by small-scale eddies could be taken into account through the inclusion of a constant eddy viscosity in (1), as in Townsend (1970). However, the results for the large-scale eddies, which are what concerns us here, would not be appreciably changed, hence viscosity is ignored.

The evolution of turbulence statistics due to purely external forcings (in this case the gradients of $\mathbf{U}$, or the effect of boundaries) is determined by adopting a spectral description of the flow (which allows elimination of the pressure perturbation) and assuming an initial energy spectrum for the turbulence. The turbulence is assumed to be locally homogeneous, and the mean velocity gradients are assumed to be locally uniform (Hunt, 1973; Durbin, 1981; Hunt et al., 1990).

A spatial scale-separation between the turbulence and the mean flow quantities is assumed, so that an average over a local volume $V(\mathbf{x})$ can be 
conceptually defined that yields, for example, the mean flow (by averaging over the turbulent part):

$$
\mathbf{U}(\mathbf{x}, t)=\frac{1}{V(\mathbf{x})} \iiint \mathbf{v}(\mathbf{x}, t) \mathrm{d} x \mathrm{~d} y \mathrm{~d} z,
$$

where $\mathbf{v}$ is total velocity, including mean and turbulent parts. Then a local turbulent spectrum can be defined, formed over the same moving-average volume, with slowly varying wavenumbers $\mathbf{k}(\mathbf{x}, t)=\left(k_{1}, k_{2}, k_{3}\right)$ and Fourier coefficients $\hat{\mathbf{u}}^{(H)}(\mathbf{k}, \mathbf{x}, t)$ of the flow:

$$
\hat{\mathbf{u}}^{(H)}=\frac{1}{(2 \pi)^{3}} \iiint \mathbf{u}^{(H)} \mathrm{e}^{-i \mathbf{k} \cdot \mathbf{x}} \mathrm{d} x \mathrm{~d} y \mathrm{~d} z .
$$

In this equation and in (3), the integration is carried out over the volume $V$ (see Hunt, 1973), and the superscript $(H)$ denotes the flow away from any boundaries. The corresponding turbulent velocity may be expressed by the inverse Fourier transform:

$$
\mathbf{u}^{(H)}=\iiint \hat{\mathbf{u}}^{(H)} \mathrm{e}^{\mathrm{ik} \cdot \mathbf{x}} \mathrm{d} k_{1} \mathrm{~d} k_{2} \mathrm{~d} k_{3}
$$

Note that both $\hat{\mathbf{u}}^{(H)}$ and $\mathbf{k}$ are assumed to be slow functions of the spatial coordinates (changing appreciably only over several wavelengths). Additionally, not only is $\hat{\mathbf{u}}^{(H)}$ a function of time, satisfying an equation that results from (1) in spectral space, but $\mathbf{k}$ also evolves in time, according to the equation (Dubrulle et al., 2004; Teixeira and Belcher, 2006)

$$
\frac{\partial \mathbf{k}}{\partial t}+\nabla(\mathbf{k} \cdot \mathbf{U})=0
$$

The approach followed here is essentially the same as described in detail in Teixeira and Belcher (2002). 
The presence of a rigid boundary acts to block the turbulence at the surface, so that the normal component of the fluctuating velocity $(w)$ is brought to zero at the interface. Following Hunt and Graham (1978) this process is represented over short times by adding to the flow within the fluid the irrotational flow induced by image vortices above the interface, namely

$$
\mathbf{u}(t=0)=\mathbf{u}^{(H)}+\nabla \phi^{(S)}(t=0) .
$$

Here $\phi^{(S)}$ is a velocity potential, associated with the image vortices induced by the interface to ensure no deformation of the interface by the turbulence. This correction remains irrotational if the distorting flow is irrotational (e.g. a surface wave). If the distorting flow possesses vorticity (e.g. shear), the correction ceases to be irrotational over time.

KST (see Turfus and Hunt, 1987; Perkins et al., 1990; Fung et al., 1992), goes one step further beyond RDT by providing actual realisations of turbulent flows. This is particularly useful for tracking the trajectories of tracer particles or calculating Lagrangian statistics. Turbulence is represented as the sum of a discrete set of Fourier modes, with a random phase added to each mode, while at the same time satisfying a given energy spectrum. The constraint of incompressibility, (2), is enforced by calculating the velocity field as the curl of another vector field, so that its divergence is zero. Hence, for example, the turbulent velocity far from any boundary is represented as

$$
\mathbf{u}^{(H)}=\sum_{n}\left[\mathbf{a}_{n} \cos \left(\mathbf{k}_{n} \cdot \mathbf{x}\right)+\mathbf{b}_{n} \sin \left(\mathbf{k}_{n} \cdot \mathbf{x}\right)\right]
$$

where

$$
\mathbf{a}_{n}=\hat{\mathbf{a}}_{n} \times \hat{\mathbf{k}}_{n}, \quad \mathbf{b}_{n}=\hat{\mathbf{b}}_{n} \times \hat{\mathbf{k}}_{n}, \quad n=1,2, \ldots
$$


are Fourier amplitudes, $\mathbf{k}_{\mathbf{n}}$ are wavenumber vectors and $n$ is the number of the mode. $\hat{\mathbf{k}}_{n}=\mathbf{k}_{n} /\left|\mathbf{k}_{n}\right|$ are normalised vectors with the same direction as the wavenumber $\mathbf{k}_{n}$ and $\hat{\mathbf{a}}_{n}$ and $\hat{\mathbf{b}}_{n}$ are vectors with the same modulus as $\mathbf{a}_{n}$ and $\mathbf{b}_{n}$, respectively. The directions of $\mathbf{k}_{n}, \hat{\mathbf{a}}_{n}$ and $\hat{\mathbf{b}}_{n}$ are picked from random distributions. Additionally, the values of $\hat{\mathbf{a}}_{n}$ and $\hat{\mathbf{b}}_{n}$ are picked from a Gaussian distribution consistent with the prescribed energy spectrum. In the calculations that follow, 300 Fourier modes, corresponding to 300 different wavenumber values and directions will be considered.

The initial state of the turbulence is taken here to be isotropic, and with the von Kármán energy spectrum,

$$
E\left(k_{0}\right)=q^{2} l \frac{g_{2}\left(k_{0} l\right)^{4}}{\left(g_{1}+\left(k_{0} l\right)^{2}\right)^{\frac{17}{6}}},
$$

where $k_{0}$ is the initial wavenumber magnitude, $g_{1}$ and $g_{2}$ are dimensionless constants, $q$ is the root-mean-square turbulent velocity and $l$ is the longitudinal length scale of the initial turbulence. In practice, this spectrum is truncated at $k_{0} l=5$ in the KST results of this section in order to realistically eliminate small scales in the turbulence and reduce noise (this mimics the effect of a finite Reynolds number - see Teixeira and Belcher, 2000).

Two types of distorting mean flow are considered here: firstly a constantshear flow aligned in the $x$-direction,

$$
U=\alpha z, \quad V=0
$$

where $\alpha$ is the shear rate. The total distortion, or total strain, to the turbulence is then characterised by the dimensionless time $\beta=\alpha t$. Secondly, we consider the distortion due to the mean velocity that corresponds to an 
irrotational wave propagating in the $x$ direction, which may be written

$$
\begin{aligned}
& U=a_{w} k_{w} c_{w} \mathrm{e}^{k_{w} z} \cos \left(k_{w} x-\sigma_{w} t\right), \\
& W=a_{w} k_{w} c_{w} \mathrm{e}^{k_{w} z} \sin \left(k_{w} x-\sigma_{w} t\right),
\end{aligned}
$$

where $a_{w}, k_{w}, c_{w}$ and $\sigma_{w}=c_{w} k_{w}$ are, respectively, the wave amplitude, wavenumber, phase speed and angular frequency. In fact, as shown by TB2002, what is relevant for the distortion of the turbulence over time intervals longer than a wave period is the vertical gradient of the Stokes drift of the wave, which is steady and given by

$$
\alpha_{S}=\frac{d U_{S}}{d z}=2\left(a_{w} k_{w}\right)^{2} \sigma_{w} \mathrm{e}^{2 k_{w} z},
$$

and for that reason the total distortion to the turbulence is characterised by the dimensionless time $\beta_{S}=\alpha_{S}$. It can be shown that the linearised wave-averaged vorticity equation that the turbulent motion must satisfy in this case is

$$
\partial \omega / \partial t+\mathbf{U}_{S} \cdot \nabla \omega=\omega \cdot \nabla \mathbf{U}_{S}
$$

where the systematic straining by the Stokes drift has been included. $\omega$ is the turbulent vorticity and $\mathbf{U}_{S}$ is the Stokes drift velocity. This equation can be obtained by taking the curl of the linearised Craik-Leibovich momentum equation containing a vortex-force, and is thus essentially equivalent to it.

The solutions for $\mathbf{k}$ and $\hat{\mathbf{u}}^{(H)}$ that result from (6) and from the equation that is obtained from inserting (5) into (1) are given, for the shear flow (11) by e.g. Lee et al. (1990) and for the wave flow (12) by TB2002.

\subsection{Shear and wave-distorted turbulence}

Before RDT and KST are compared quantitatively with LES of Langmuir turbulence, the structure of shear turbulence and turbulence distorted by an 
irrotational surface wave will be contrasted.

Fig. 1 shows cross-sections, at a depth $z / l=10$, of the streamwise $(u)$, spanwise $(v)$ and vertical $(w)$ velocity fluctuations for turbulence distorted by the constant-shear flow (11), after being distorted by this flow for a dimensionless time $\beta=5$. This value is used for purely illustrative purposes, since it allows a distinctive turbulence anisotropy to develop, being nevertheless not too different from values of the same parameter used in other RDT studies (e.g. Lee et al., 1990; Mann, 1994).

An air-water interface is assumed to exist at $z=0$ and the Froude number of the turbulence is assumed to be so low that the turbulence does not deform the interface. The depth chosen is such that influence from the boundary is insignificant. As can be seen, $v$ and especially $u$ have large magnitude, while $w$ is considerably weaker. The ordering of the magnitudes of the velocity fluctuations is $\overline{u^{2}}>\overline{v^{2}}>\overline{w^{2}}$, and the $u$ velocity fluctuations are also elongated along $x$, as is typical of shear-driven boundary layers. This flow structure is similar to that presented in the RDT study of Lee et al. (1990) and in the direct numerical simulations (DNS) of Tsai et al. (2005).

Fig. 2 shows cross-sections of the components of the velocity fluctuations for turbulence distorted by a surface wave, as in TB2002, (12). In Fig. 2, a wave of slope $a_{k} k_{w}=0.2$ and a time normalised by the wave period of $t / T=10$ has been assumed (as in TB2002), so that the dimensionless time is $\beta_{S}=5$, similarly to Fig. $1 . k_{w} l$ has also been assumed to be zero, which ensures that $\alpha_{S}$ is effectively constant with depth (as $\alpha$ was in Fig. 1). This is done here to facilitate the comparison, but would correspond in reality to very long surface waves or very small-scale turbulence. 
While, as was seen, the total strain imposed on the turbulence in Fig. 2 is the same as in Fig. 1, its effects are totally different. In Fig. 2, the $u$ velocity fluctuations are weak, while the magnitude of both $v$ and $w$ is large. The appearance of the turbulent velocity fluctuations is consistent with an ordering of the variances $\overline{u^{2}} \ll \overline{v^{2}} \approx \overline{w^{2}}$ (cf. Li et al. 2005), and the flow structure, particularly that of $w$, is strongly elongated in the $x$ direction. The behaviour of the $w$ velocity field is in qualitative agreement with results of Skyllingstad and Denbo (1995, plates 3, 6 and 9), MSM97 (Figs. 12, 13 and 22), and Noh and Min (2004, Fig. 1), although streamwise mergers, which appear to be intrinsically of nonlinear origin, are mostly absent. This ability of RDT to produce streamwise vortices in Langmuir turbulence mirrors its ability to produce elongated structures of high $u$ fluctuations (streaky structures) in turbulence subjected to mean shear (Lee et al., 1990). This suggests that linear processes are responsible for the appearance of both structures.

In Fig. 3, the RDT results of TB2002 for the evolution of the velocity variances as a function of dimensionless time, where the mean velocity (12) is used, are compared with RDT results where the rectified equation (14) is used instead. As can be seen, the average evolution is the same, only differing in the superposed oscillations, associated with distortion of turbulence by the individual wave cycles. For that reason, the second, simpler approach is adopted throughout the remainder of this paper.

An aspect that was overlooked in the study of TB2002 is how the turbulence energy is distributed among the different scales of motion. This question may be addressed by calculating 1D wavenumber spectra of turbulence, 
which are defined as:

$$
\begin{aligned}
& S_{i i}\left(k_{1}\right)=\frac{1}{2 \pi} \int \overline{u_{i}(x, y, z) u_{i}\left(x+r_{1}, y, z\right)} \mathrm{e}^{-i k_{1} r_{1}} d r_{1} \\
& S_{i i}\left(k_{2}\right)=\frac{1}{2 \pi} \int \overline{u_{i}(x, y, z) u_{i}\left(x, y+r_{2}, z\right)} \mathrm{e}^{-i k_{2} r_{2}} d r_{2}, \quad(i=1,2,3) .
\end{aligned}
$$

Results from RDT presented in Fig. 4 show that, in shear turbulence the energy of streamwise velocity fluctuations is increased at all scales in the streamwise direction, while a peak develops at the integral length scale in the spanwise direction. This is consistent with a 'streaky structure' of $u$. Under a Stokes distortion, it is the vertical velocity fluctuations that are increased: on a broad range of scales in the streamwise direction, and with a peak in the spanwise direction. The main difference between shear and wave-distorted turbulence in the spanwise fluctuations is that in the shear turbulence the increase in $v$ energy occurs primarily at small scales, while in wave-distorted turbulence it occurs at all scales. These results essentially confirm the interpretation of TB2002 based on the behaviour of the integral length scales in shear and wave-distorted turbulence.

\subsubsection{Blocking effect of the free surface}

The Froude number of turbulence in the water is often sufficiently low that the shape of the free surface bounding it above can be taken as specified and fixed. This shape can either be flat (in the absence of surface waves, when the distorting flow is a shear flow) or undulating (when the distorting flow is an irrotational wave). The undulation associated with the wave may be treated rigorously using curvilinear coordinates (TB2002). However, when the slope of the distorting wave is small, these coordinates essentially coincide with the Cartesian coordinates, and the air-water interface is also approximately 
flat. In either of these two situations, the presence of the interface acts to block the turbulence at the surface, so that the normal component of the fluctuating vorticity is brought to zero at the interface.

The effect of blocking on isotropic turbulence is well-known (Hunt and Graham, 1978; Hunt, 1984), resulting (in the RDT approximation) in an amplification of the tangential velocity components of the turbulence at the surface by a factor of 1.5, while the normal component tends to zero. For a constant-shear flow, Fig. 5 shows that the blocking effect suppresses almost completely the streaky structures at the surface, making the $u$ and $v$ field appear almost isotropic along $x$ and $y$ (cf. Lee and Hunt, 1989; Mann, 1994). This phenomenon, which is related to the generation of vorticity in the blocking velocity correction (see Gartshore et al., 1983), would have important consequences for surface transport if true in realistic conditions. However, it is not supported by the DNS results of Tsai et al. (2005), where streaky structures are seen to exist up to the surface. There are a number of possible reasons for this disagreement. The assumption of initial irrotationality of the blocking correction, or of a unique, constant, shear rate and length scale, may not be appropriate, since in the DNS of Tsai et al. (2005) the turbulence spreads from the free surface (through a surface stress) instead of impacting on the interface from below, as implied by RDT.

In Fig. 6, the velocity field at the surface in turbulence distorted by a surface wave is displayed. Here, both $u$ and $v$ are somewhat larger than in the bulk of the fluid, but behave in qualitatively the same way, with $v$ being much larger than $u$, and showing a structure elongated in the $x$ direction. Obviously, this has important consequences for transport at the surface, as 
will be seen next. By Hunt and Graham's (1978) results (which hold in the present case), the TKE must be the same in the bulk of the fluid and at the surface. Thus, for turbulence distorted by a surface wave, $v$ is amplified by a factor larger than $1.5(\approx 2)$ due to blocking, since $u$ is relatively small in the bulk of the fluid, but $w$ must decay to zero at the surface, transferring the whole of its energy to $v$ in the process.

\subsection{Surface transport of tracer particles}

Fig. 7 shows the locations of 10000 tracer particles released randomly at the surface for each of the flows considered. Fig. 7a displays the initial particle positions. Figs. $7 \mathrm{~b}$ and $7 \mathrm{c}$ display the particle spatial distributions after an advection time $q t / l=1$ (corresponding to one eddy turn-over time of the initial turbulence), respectively for shear turbulence and turbulence distorted by a wave. It can be seen that the tracer particles tend to accumulate in convergence zones of the surface velocity field, forming rows. For turbulence distorted by a wave the particles accumulate in well-defined rows clearly aligned in the streamwise direction. Given the nature and intensity of $v$ in this last case (see Fig. 6b), that behaviour is not surprising, and is clearly in qualitative agreement with that observed in the LES of Skyllingstad and Denbo (1995, plate 6), MSM97 (Fig. 10), McWilliams and Sullivan (2001, Fig. 2) and Skyllingstad (2001, Figs. 2 and 3). It also resembles the behaviour of surface tracers in the experimental studies of Faller and Cartwright (1983), Nepf et al. (1995) and Melville et al. (1998). Taking as an example for comparison Fig. 10 of MSM97, the time indicated in that figure is $t=1440 \mathrm{~s}$. If the value of the integral length scale found later in Section 3.4 is adopted, $l=7.5 \mathrm{~m}$, and the normalised shear stress is calcu- 
lated using $\mathrm{RDT}$ for $\beta_{S}=5$ (the dimensionless time used to compute the velocity field that advects the tracer in Fig. 7), this gives $\overline{u w} / q^{2}=-0.7$. Then $q=1.2 u_{*}$, and using the value $u_{*}=6.1 \times 10^{-3} \mathrm{~m} \mathrm{~s}^{-1}$ given by MSM97, in Fig. 10 of MSM97 the advection time is $q t / l=1.4$. Despite the many differences between the two models, it is reassuring that this is at least of the same order of magnitude as the advection time adopted in Fig. 7.

In the case of shear turbulence (Fig. $7 \mathrm{~b}$ ), the tracer rows are not so well defined as in Fig. 7a, and have a more isotropic distribution, although there is the hint of alignment in the streamwise direction. This is due to the weakness of the associated advecting velocity field, mentioned above. The DNS of shear turbulence near an interface computed by Tsai et al. (2005) does have streaky structures at the surface and their tracer particles tend to form much clearer rows in the streamwise direction than in Fig. 7b. However, even if such streaks do exist, a flow whose dominant velocity fluctuation is $u$ with an elongated structure along $x$ is, at least intuitively, less effective in creating streamwise tracer rows than a flow with convergence zones along $x$ in the $v$ velocity field. Fig. 8 shows schematically the different mechanisms for the formation of tracer rows. While in shear flow these rows occur due to the confluence of $v$ at the entrance of the jet of high $u$, in a flow with streamwise vortices the tracer rows are formed by the lines of strong convergence of the $v$ velocity field.

It has additionally been noted by Craik and Leibovich (1976), Leibovich (1983), Cox and Leibovich (1993) and Thorpe (2004) that particles in these streamwise rows of tracer travel in the direction of the mean flow faster than the air-water interface itself, on average. In the context of turbulence, 
this is easily explained by the existence of a shear stress. Since the tracer accumulates above zones where the flow descends, in order to have a negative shear stress near the surface, the $u$ velocity perturbation must be positive there. This argument, which also applies to shear turbulence, explains the analogous behaviour of tracers in the DNS of Tsai et al. (2005). In the case of wave-distorted turbulence, the tracer particles in our KST moved in the direction of the wave propagation (or of the Stokes drift) with a velocity exceeding the average interface velocity by $0.45 q$, a rather significant value.

These results illustrate the differences between turbulence in a shear flow and turbulence distorted by a wave. A simple explanation of the differences in terms of the dynamics of the vorticity is described in Appendix A. The results of this section show that at least the qualitative aspects are captured by the linear RDT and KST models. In the next two sections quantitative comparisons are made between these models and LES of Langmuir turbulence.

\section{Scaling the large-scale structure of Langmuir turbulence}

Polton and Belcher (2007) investigate the TKE budget of their LES of Langmuir turbulence and show that in an upper Stokes layer, whose depth scales on the depth of the Stokes drift, the dominant balance is between production of TKE by the Stokes production, and dissipation (in the absence of wave breaking). Below this region turbulent transport carries TKE downwards deep into the mixed layer. They then suggest a schematic where, within the Stokes layer, the Stokes drift tilts and stretches vertical vorticity into the horizontal. This generates convergence zones, which then leads 
to downwelling jets that penetrate deep into the mixed layer (and transport TKE through turbulent transport). We consider now whether or not the linear RDT model can capture quantitatively the structure of Langmuir turbulence computed in the LES, particularly in the Stokes layer.

Grant and Belcher (2009) have used the TKE budget to develop a scaling for the resulting Langmuir turbulence, arguing that the appropriate velocity scale is $w_{* L}=\left(u_{*}^{2} U_{S 0}\right)^{\frac{1}{3}}$ (see also Harcourt and D'Asaro, 2008) and the appropriate length scale is $h$, the depth of the mixed layer. They show that profiles of the turbulent velocity variances from a wide range of simulations, when scaled by $w_{* L}^{2}$ and $h$, collapse onto single profiles. It is then sufficient to consider results from a single simulation, and the focus here is on the shape of the profiles of the velocity variances.

We focus on the LES run E/0.3 of Langmuir turbulence computed by MSM97. Subsequent investigations (Li et al., 2005) have suggested that the value of the turbulent Langmuir number used by MSM97 in this experiment is fairly typical of ocean conditions. MSM97 consider a monochromatic wave of amplitude $a_{w}=0.8 \mathrm{~m}$ and wavenumber $k_{w}=2 \pi / 60 \mathrm{rad} \mathrm{m}^{-1}$, so that the wave slope is $a_{w} k_{w}=0.084$, and the angular frequency, obtained using the linear dispersion relation of surface water waves, is $\sigma_{w}=1.0 \mathrm{rad} \mathrm{s}^{-1}$. In addition, they specified a surface wind stress with an associated friction velocity in the water of $u_{*}=6.1 \times 10^{-3} \mathrm{~m} \mathrm{~s}^{-1}$, and a thermocline at $h=33 \mathrm{~m}$. These values will be used here. For this simulation the Stokes layer occupies the region $0<z / h<0.4$, a substantial fraction of the mixed layer (Grant and Belcher, 2009). 


\subsection{Deformation by shear and Stokes drift}

Turbulence in the LES simulations of the wind-driven mixed layer is subjected to straining from three sources: the presence of the air-sea interface; the mean shear in the wind-driven current; and the Stokes drift associated with the surface wave. Consider first the competing strains of the mean shear and the Stokes drift, which can be measured by the parameter $R$, defined by

$$
R=\frac{\alpha_{S}}{\alpha}
$$

This parameter gives the ratio of the production of TKE by the Stokes drift and by shear, according to Equation (5.1) of MSM97. The variation of this parameter is calculated next using results from the LES of MSM97.

Fig. 9a shows the variation with depth of the strain rate associated with the Stokes drift of the wave (dashed line), derived from the parameters given by MSM97. Also shown in Fig. 9a is the shear rate through the wind-driven mixed layer derived from the mean velocity profiles computed by MSM97 for simulations with and without Stokes drift. Both cases are driven by a surface wind stress, with an associated friction velocity (in the water) equal to $u_{*}=6.1 \times 10^{-3} \mathrm{~m} \mathrm{~s}^{-1}$. The case without Stokes drift, $\mathrm{S} / \infty$ (dotted line), shows a shear rate that closely follows the form $\alpha=u_{*} / \kappa z$ expected for a logarithmic surface layer with the appropriate friction velocity. The shear rate in the case with Stokes drift, E/0.3 (solid line), perhaps surprisingly, also approximately follows the form expected for a logarithmic surface layer, but this time with a much reduced friction velocity of $0.61 \times 10^{-3} \mathrm{~m} \mathrm{~s}^{-1}$ (see Fig. 9a). The reason is the following: once Langmuir turbulence is initiated, mixing is promoted by the Langmuir circulations themselves reducing the 
mean shear, perhaps augmented by the effects of the Coriolis force turning the mean flow and reducing shear in the wave direction (see Polton et al., 2005; Polton and Belcher, 2007). The assumption of a logarithmic mean velocity profile, employed above, is an approximation primarily valid near the surface, since, for example, Fig. 3a of MSM97 shows that the shear stress in the $x$ direction is not constant, but decreases with depth. However, as the following scalings rely primarily on the flow parameters near the surface, say for $z<0.4 h$, where the mean transport gradients are sufficiently strong, this approximation is accurate enough for our purposes.

With these observations the parameter $R$ for a single wave can be written

$$
R=\frac{2\left(a_{w} k_{w}\right)^{2} \sigma_{w} e^{-2 k_{w} z}}{u_{* s} / \kappa z}
$$

where $u_{* s}$ is an effective friction velocity associated with the near-surface shear. Fig. 9b shows the variation of $R^{-1}$ with depth for run $\mathrm{E} / 0.3$, when $u_{* s}=0.61 \times 10^{-3} \mathrm{~m} \mathrm{~s}^{-1}$ (solid line), together with values obtained from the LES profiles (symbols). Also shown is the profile obtained using the full friction velocity, $u_{*}=6.1 \times 10^{-3} \mathrm{~m} \mathrm{~s}^{-1}$ (dotted line). When the mean shear is correctly parameterised using $u_{* s}$, then $R^{-1} \ll 1$ through most depths, implying the strain by Stokes drift is greater than the strain by mean shear. Towards the bottom of the wind-driven layer, $0.8<z / h<1, R<1$ but at these depths strains by both Stokes drift and shear are weak, and the turbulence is likely to be dominated by entrainment at the thermocline.

We conclude that in the presence of Stokes drift the turbulence is largely distorted by Stokes drift because this situation is self-sustained by intense vertical mixing. While turbulence can mix down momentum, it has no impact on the Stokes drift gradient. The deformation by mean shear on the 
turbulence can therefore be neglected.

\subsection{Integral properties of Langmuir turbulence}

The next step is to evaluate two integral properties of Langmuir turbulence, which will then enable scaling arguments. Firstly, an estimate of the integral length scale of the Langmuir turbulence computed by MSM97 is evaluated using the approximation used in the $K-\varepsilon$ turbulence closure (although we note that this is only strictly valid for homogeneous and isotropic turbulence), namely

$$
l \propto \frac{K^{\frac{3}{2}}}{\varepsilon} \Rightarrow \frac{l}{h}=c_{1} \frac{\left(K / u_{*}^{2}\right)^{\frac{3}{2}}}{\varepsilon h / u_{*}^{3}},
$$

where $K$ is the TKE, $\varepsilon$ is the rate of viscous dissipation and $c_{1}$ is a dimensionless constant of $O(1)$. For homogeneous turbulence, the relation between the dissipation rate $\varepsilon$, the turbulent root-mean square velocity $q$ and the longitudinal length scale $\varepsilon \propto q^{3} / l$ has been found by various authors (Pearson et al., 2002; Kaneda et al., 2003) to have a proportionality constant approximately between 0.5 and 1 . In terms of (18), this would mean that $c_{1}$ should be between 0.27 and 0.54 . Here we choose $c_{1}=0.387$, which gives optimal agreement between the RDT calculations and the LES data, as will be seen below.

Figs. 4 and 5 of MSM97 present profiles of the normalised TKE, $K / u_{*}^{2}$, and of the normalised dissipation rate $\varepsilon h / u_{*}^{3}$. Fig. 10a shows a profile of $l / h$, as defined in (18), derived from these LES data. It can be seen that the integral length scale of the turbulence increases approximately linearly away from the boundary. The dashed line in Fig. 10a represents a linear fit to the 
LES data, namely

$$
l / h=\gamma\left(z+d_{l}\right) / h
$$

The best fit yields a slope $\gamma \approx 0.35$ and a value at the surface, akin to the displacement height of a logarithmic layer, $d_{l} / h \approx 0.42$. A linear variation in the integral length scale is a characteristic of either a shear-free turbulent layer near an interface, which has $\gamma \approx 1$ (Hunt 1984), or a constant-stress logarithmic boundary layer, which has $\gamma=\kappa \approx 0.4$, the von Kármán constant (Tennekes and Lumley, 1972). The latter value is surprisingly close to the value of $\gamma$ obtained here for the Langmuir turbulence.

We note that the value of the displacement height obtained from the LES may be an artifact of the way the LES resolves the interface. Nevertheless a non-zero value is probably realistic because the turbulence length scale is determined by non-local factors. So it does not tend to zero at the surface, because eddies of finite size approach the surface where they are blocked by the air-water interface (Hunt, 1984). We note that the value of $\gamma$ derived by Grant and Belcher (2009) in their Fig. 6 is considerably larger that the value found here. But they force their linear fit to intercept the origin, making their value larger. A consistency check to our choice of $c_{1}$ is that if the integral length scale is everywhere smaller than $l / h=1$, then $l / h$ should grow linearly until very close to $z / h=1$, instead of tending to a constant value, as in Grant and Belcher (2009). This is indeed confirmed by Fig. 10.

Secondly, the decorrelation time scale, or eddy turn-over time of the turbulence, $T_{e}$, is estimated by analogy with the integral length scale, namely

$$
T_{e} \propto \frac{K}{\varepsilon} \Rightarrow \frac{T_{e} u_{*}}{h}=\frac{K / u_{*}^{2}}{\varepsilon h / u_{*}^{3}} .
$$


Note that in (20), and in contrast to (18), we have not included a constant of proportionality, because the eddy turn-over time is generally not as precisely defined in terms of the other quantities as the integral length scale, and also because it is the form of its dependence on depth that will be of primary interest for the RDT and KST calculations.

Fig. 10b shows the variation of $u_{*} T_{e} / h$ with depth computed from the LES data of MSM97. The decorrelation time increases approximately linearly with distance from the boundary, and can be fitted to

$$
\frac{u_{*} T_{e}}{h}=\delta\left(z+d_{T}\right) / h,
$$

with slope $\delta \approx 1.0$ and displacement height $d_{T} / h \approx 0.08$. This fit is shown as the solid line in Fig. 10b. A linear increase in $T_{e}$ with distance from a boundary is also a characteristic of shear-free turbulence near a boundary, where $\delta \approx 1$, and a constant-stress logarithmic boundary layer, where $\delta=\kappa$ (Tennekes and Lumley, 1972). The variation of the decorrelation time scale in Langmuir turbulence is therefore similar to the variation in wall-bounded shear-free turbulence.

In the simulations of MSM97 $u_{*}=6.1 \times 10^{-3} \mathrm{~m} \mathrm{~s}^{-1}$, whence the decorrelation time scale at the interface is $T_{e 0}=\delta d_{T} / u_{*} \approx 430 \mathrm{~s}$, which is again non-zero because $T_{e}$ receives contributions from eddies of finite size that reach the surface from some distance below.

These two measures of the turbulence both increase away from the boundary and so indicate the importance of the air-water interface in blocking the turbulence. The behaviour of the integral length and time scales will prove useful in estimating the nonlinear processes in the Langmuir turbulence, which is done next. 


\subsection{Scaling the distortion of the turbulence}

Consider now the velocity fluctuations in the Langmuir turbulence, $q$, which scale on the friction velocity, $u_{*}$, (MSM97). The velocity associated with the deformation of the turbulence is the Stokes drift, $U_{S}=\left(a_{w} k_{w}\right)^{2} c_{w} \mathrm{e}^{-2 k_{w} z}$. At the air-sea interface the ratio of these terms is

$$
U_{S} / q \sim\left(a_{w} k_{w}\right)^{2} c_{w} / u_{*}
$$

For the parameters of run E/0.3 in MSM97, this ratio is approximately equal to 11-a large number. Similarly, the fluctuating strain rate associated with the turbulence can be estimated to be $q / l$ which scales as $T_{e}^{-1}$, whereas the strain rate associated with the Stokes drift is $\alpha_{S}$. Using the expression obtained from the LES in Section 3.2 for $T_{e}$, the ratio of these terms is

$$
\frac{\alpha_{S}}{T_{e}^{-1}} \sim 2 \frac{c_{w}}{u_{*}}\left(a_{w} k_{w}\right)^{2} \delta k_{w}\left(z+d_{T}\right) e^{-2 k_{w} z}
$$

The maximum value of this ratio occurs at $2 k_{w}\left(z+d_{T}\right)=1$, when it takes the value

$$
\left.\frac{\alpha_{S}}{T_{e}^{-1}}\right|_{\max } \sim \frac{c_{w}}{u_{*}}\left(a_{w} k_{w}\right)^{2} \delta \exp \left(2 k_{w} d_{T}-1\right),
$$

which, for case E/0.3 of MSM97, equals about 7-again a relatively large number. Hence for this case at least the fluctuating turbulent velocity and strain rates are much smaller than the velocity and strain rate associated with the deformation due to the Stokes drift. This proves to be a key in justifying a linearised RDT model for Langmuir turbulence in the Stokes layer.

Finally, we note that the Froude number associated with the turbulent motions is large, so that, as assumed in the LES, the turbulence does not 
appreciably deform the interface, i.e.

$$
a_{w} \gg q^{2} / \mathrm{g}
$$

where $g$ is the acceleration of gravity. Hence the interface remains dominated by the driving wave (cf. Brocchini and Peregrine, 2001).

\subsection{Estimating the parameters of the RDT model}

RDT is, mathematically, an initial-value problem and so requires specification of the initial turbulence, and then specification of the integration time of the distortion (or equivalently the total strain by the mean flow).

The initial turbulence is represented by the spectrum (10), which requires specification of the integral length scale, $l$, and turbulent intensity, $q$. Here, we will not need to specify $q$ because velocity variances will be normalised on $q$. Turbulence statistics will be shown here as a function of normalised distance from the boundary, $z / l$, but the results of MSM97 are plotted as a function of $z / h$. Hence we require a relation between $l$ and $h$. Within the RDT model the principal effect of the integral length scale is to determine the depth of the blocking effect of the air-sea interface on the turbulence, and hence we relate $l$ and $h$ here to match this blocking depth. The variation in $l$ with $z$ obtained in Section 3.2 from the LES data shows that far from the interface $l<z$, and hence the turbulence at these depths is unaffected by the boundary, whereas near the interface $l>z$, and so the turbulence there is directly affected by the blocking of the boundary. Hence in the RDT model we use the value of the $l$ obtained from the LES at the depth where $l=z$, i.e. the intersection between the line $y=l(z)$ in Fig. 10a and the line $y=z$. The turbulence is then subjected to blocking over the correct distance from 
the boundary. For the parameters of the MSM97 simulations, this procedure yields $l / h=0.23$, so that $l=7.5 \mathrm{~m}$.

Now we turn to the distortion time. As stated above, if the linearisation assumption in RDT model is to be self-consistent, the distortion time $T_{d}$ must be a fraction of the eddy turn-over time $T_{e}$. Hence it seems natural to assume a simple proportionality

$$
T_{d}(z)=\mu T_{e}(z)
$$

where $\mu$ is a constant of $O(1)$. We conceive that the Stokes drift deforms the turbulence over some fraction of the decorrelation time and then nonlinear processes arrest the deformation. A statistically steady state is achieved with eddies born, distorted by the Stokes drift over a fraction of their lifetime and then dying. Since the decorrelation time scale increases with depth, so then does the time over which the Stokes drift deforms the turbulence. The value of $\mu$ is determined by comparison between the RDT model and the LES data (Fig. 12 in Section 4), yielding a value of $\mu=0.43$, which is of $O(1)$, as expected. The use of a depth-dependent time is in a sense analogous to the approach employed in Mann's (1994) RDT study of boundary-layer turbulence, where the model time is scale-dependent.

Equation (26) gives a dimensional time. To obtain the corresponding dimensionless time $\beta_{S}, T_{d}(z)$ must of be multiplied by $\alpha_{S}(z)$, consistent with the values of the wave parameters of MSM97, namely

$$
\beta_{S}=2\left(a_{w} k_{w}\right)^{2} \sigma_{w} e^{-2 k_{w} z} \mu T_{e}(z)
$$

The variation of $\beta_{S}$ given by (27) is shown in Fig. 11 as the solid line. Notice that the distortion is largest within the Stokes layer $(0<z / h<0.4)$. The 
dimensionless distortion time attains a maximum slightly above 3 near the surface, but decays to zero with the Stokes drift as depth increases. This is roughly consistent with the values of approximately 2 to 3 assumed in numerous RDT studies (e.g. Townsend, 1970; Mann, 1994). Also shown in Fig. 11 is the dimensionless time that would be obtained if the eddy turnover time had its surface value $T_{e}(z=0)$ everywhere. In this case $\beta_{S}$ is smaller, and exactly proportional to the Stokes drift strain rate.

A final comment is due. The LES of MSM97 use horizontal grid spacings of $\Delta x=3 \mathrm{~m}$ and $\Delta x=4.7 \mathrm{~m}$ in experiments $S / \infty$ and $E / 0.3$, respectively, and a vertical grid spacing of $\Delta z=0.6 \mathrm{~m}$. This effectively limits the wavenumbers that may be present in the LES turbulence spectrum. In particular, for the quoted cases, the dimensionless wavenumber in (10) is smaller than $k_{0} l=7.8$ or $k_{0} l=5.0$ in the horizontal, respectively, while the vertical wavenumber is smaller than $k_{0} l=39.4$. This anisotropy is not taken into account in the spectral approach of RDT and KST so, since we are going to focus primarily on Langmuir turbulence (experiment $E / 0.3$ ) the spectrum (10) is truncated at $k_{0} l=5$ in the calculations that follow, as was done in Section 2 without the present justifications.

\section{Comparison between RDT and LES results}

Results calculated with RDT and KST using the previously estimated parameters are now compared with those computed by MSM97 in their LES. Firstly statistics of the distorted turbulence are calculated to show how the profiles are shaped by the combination of Stokes drift, blocking by the interface, and variation of the turbulence scale with distance from the interface, 
modelled here by allowing $T_{e}$ to vary with depth. Secondly, a realisation of the turbulent flow similar to that presented in Section 2, but for the specific conditions considered in MSM97 is calculated using KST.

\subsection{Profiles of the turbulent velocity variances}

Fig. 12a shows the turbulent velocity variances normalised on TKE, $\overline{u_{i}^{2}} / \frac{2}{3} K$, calculated from RDT and comparisons with the LES data presented in Fig. 6 of MSM97 for run E/0.3. Note that RDT is an initial-value problem, so it is appropriate to compare the ratios of the turbulence intensities, but not their actual values, since these are dependent on the initial conditions.

The RDT results in Fig. 12a agree remarkably well with the LES data, particularly in the Stokes layer $0<z / h<0.4$. Deep in the mixed layer, for $z / h$ larger than about 0.7 the variances are approximately isotropic (when the normalised variance is 1 by definition). $\left(\overline{w^{2}} / \frac{2}{3} K\right.$ is slightly smallerprobably a consequence of the thermocline at $z / h=1$ in the LES.) Nearer the surface, in $0.2<z / h<0.7$, the streamwise variance, $\overline{u^{2}} / \frac{2}{3} K$, decreases, while $\overline{v^{2}} / \frac{2}{3} K$ and $\overline{w^{2}} / \frac{2}{3} K$ both increase towards the interface. By $z / h=0.2$, $\overline{v^{2}} / \frac{2}{3} K$ and $\overline{w^{2}} / \frac{2}{3} K$ are considerably larger than $\overline{u^{2}} / \frac{2}{3} K$. This behaviour is consistent with the generation of streamwise vortices by the tilting and stretching of vertical vorticity into the streamwise direction by the Stokes drift (TB2002). In the region $0<z / h<0.2, \overline{v^{2}} / \frac{2}{3} K$ and $\overline{u^{2}} / \frac{2}{3} K$ increase towards the interface, while $\overline{w^{2}} / \frac{2}{3} K$ is forced to decrease to zero. This region

corresponds to $z / l<1$ and so is caused by the blocking effect of the interface on the turbulence distorted by the Stokes drift.

Consider now how different parts of the RDT solution give different parts of the response. Fig. 12b presents profiles of the turbulent velocity variances 
for the same conditions as in Fig. 12a, except that the deformation is allowed for the same time through the whole depth of the layer (dotted line in Fig. 11). Hence the model is truncated after a dimensionless distortion time $\beta_{S}$ corresponding to the eddy turn-over time valid at the surface through all depths. Although the RDT values near the surface are close to the data, the anisotropy due to distortion by the wave motion decays too fast away from the interface, because the distortion by the Stokes drift at large depths is not allowed to act for a sufficiently long interval of time.

If, on the other hand, the Stokes drift distortion is neglected altogether and only the blocking effect of the interface is taken into account then RDT yields the results shown in Fig. 12c. Both $\overline{u^{2}} / \frac{2}{3} K$ and $\overline{v^{2}} / \frac{2}{3} K$ now increase towards the interface (by the same amount since the deformation is now isotropic in the horizontal) and $\overline{w^{2}} / \frac{2}{3} K$ decreases by the blocking mechanism towards the interface. But the amplification of $\overline{v^{2}} / \frac{2}{3} K$ and $\overline{w^{2}} / \frac{2}{3} K$ and the attenuation of $\overline{u^{2}} / \frac{2}{3} K$ farther from the interface is not produced.

The agreement between the RDT model and the LES data is better in the upper layer, $0<z / h<0.4$, which corresponds to the Stokes layer. This is consistent with the findings of Polton and Belcher (2007) and Grant and Belcher (2009) that within this upper Stokes layer the dominant balance in the TKE budget is between Stokes production and dissipation, whereas deeper in the layer turbulent transport (which is nonlinear and so not captured in RDT) is a dominant term in the TKE budget. Finally, we recall that deformation by shear would produce a completely different structure with $\overline{u^{2}}>\overline{v^{2}}>\overline{w^{2}}$, as was shown earlier in this paper, and also in TB2002. We conclude that linear processes to a large extent shape the anisotropy of 
the turbulence.

\subsection{The turbulent velocity field}

KST is now used to compute a realisation of the turbulent flow consistent with the velocity variances displayed in Fig. 12. Cross-sections of the resulting velocity field may be compared with the corresponding LES results of MSM97, displayed in their Fig. 12. In this calculation, as in Section 2, 300 Fourier modes were employed. All other parameters were kept the same as in the previous section.

Fig. 13 shows horizontal cross-sections of the instantaneous turbulent velocity field at a depth $z / l=0.40$, corresponding to the dimensional depth $z=3 \mathrm{~m}$ used in Fig. 12 of MSM97. Distances are normalised by the initial integral length scale of the turbulence $l$, but the domain has been scaled and the spacing of the contours has been chosen so as to give an appearance as close as possible to that of Fig. 12 of MSM97. The limits of normalised $x$ and $y, 40$, correspond to a dimensional distance of $301 \mathrm{~m}$, very close to that shown in Fig. 12 of MSM97.

Positive values of the velocity perturbation correspond to dark shaded contours and negative values to light shaded contours. The contour spacings in Fig. 13 were objectively made equivalent to those of MSM97 in the following way. The value of each contour used by MSM97 was divided by the square root of the TKE (taken from their Fig. 4) at the appropriate depth $z=3 \mathrm{~m}$. Then this was multiplied by $K^{1 / 2} / q$ given by RDT at the same depth. This provided velocity contours normalised by $q$, which have the same scaling as the velocity fields provided by KST. The values of these contours are used in Fig. 13. 
It can be seen that the $u$ velocity fluctuations are relatively weak and decorrelate over a large distance. The $v$ and the $w$ velocity fluctuations are more intense and the spatial structure of the $w$ velocity fluctuations reveals a compression in the $y$ direction and an elongation in the $x$ direction. As noted in Fig. 2, this spatial structure is the signature of intense and elongated streamwise vortices akin to Langmuir circulations. There is a striking similarity between Fig. 13 and Fig. 12 of MSM97, especially for the $w$ fluctuations, but also somewhat for the $u$ field. The agreement of $v$ is a little worse, with the present calculations not producing sufficient streamwise elongation. Anyway, it is surprising that KST, with its linearising assumptions, is able to reproduce so many features of this fully nonlinear turbulent flow.

\section{Conclusions}

The linearised dynamics encapsulated in rapid distortion theory and kinematic simulation of turbulence were used to understand differences between shear turbulence and Langmuir turbulence in the ocean mixed layer. In the case of turbulence distorted by a mean shear, there is a cancellation in the linearised dynamics between distortion of the turbulent vorticity by the mean flow and distortion of the mean vorticity by the turbulent flow. Consequently streamwise vorticity is not produced by mean shear. Instead the main effect is a generation of vertical vorticity that leads to the streaky structures that are widely observed in shear flows. The velocity variances are then ordered as $\overline{u^{2}}>\overline{v^{2}}>\overline{w^{2}}$. In the case of turbulence distorted by Stokes drift the cancellation no longer occurs, because the Stokes drift does not have mean vorticity. The result is that vertical vorticity is tilted into the hori- 
zontal to form streamwise vortices. The velocity variances are then ordered as $\overline{u^{2}} \ll \overline{v^{2}} \approx \overline{w^{2}}$. These qualitative results suggested that the important processes in Langmuir turbulence are controlled by linear dynamics.

These qualitative findings motivated a quantitative model for the turbulence velocity variances computed in Langmuir turbulence based on linearised RDT. Since Grant and Belcher (2009) have demonstrated that, when appropriately scaled, the profiles of turbulence variances collapse onto a single profile, it is sufficient for the RDT model to be compared with a single case of the LES model. Consequently, we used the data from an LES run by MSM97 to demonstrate that the formal approximations made in the linearised RDT model are satisfied in the LES. In particular the scalings demonstrate that in Langmuir turbulence the Stokes drift is a more potent force for distortion of turbulence than is either the mean shear or the turbulent velocity fluctuations themselves. The reason presumably is that the enhanced vertical mixing by the Langmuir circulation mixes out the mean shear, but leaves the Stokes drift unaffected. Consequently we developed here a quantitative linearised RDT model for Langmuir turbulence that includes (i) deformation of turbulent vorticity by the Stokes drift, (ii) blocking of vertical velocity fluctuations by the air-sea interface and (iii) a distortion time that increases with depth reflecting the increase of the eddy decorrelation time with depth found in LES data.

Vertical profiles of the turbulent velocity variances calculated with the linear RDT model are found to be in good agreement with the fully nonlinear LES of MSM97 particularly in the Stokes layer, which in this case occupies $0<z / h<0.4$ (Grant and Belcher, 2009). The physical picture that emerges, 
at least in the LES, is as follows. Early in the life cycle of a Langmuir eddy initial turbulent disturbances of vertical vorticity are amplified algebraically by the Stokes drift into elongated streamwise vortices, the Langmuir eddies. The turbulence is thus in a near two-component state, with $\overline{u^{2}}$ suppressed and $\overline{v^{2}} \approx \overline{w^{2}}$. Near the surface, over a depth of order the integral length scale of the turbulence, the vertical velocity is brought to zero, by blocking of the air-sea interface. Since, the turbulence is nearly two-component the energy has to go into the spanwise fluctuations, enhancing $\overline{v^{2}}$ at the interface. After a time of order half the eddy decorrelation time the nonlinear processes, such as distortion by the strain field of the surrounding eddies, arrest the deformation and the Langmuir eddy decays. The Langmuir turbulence then consists of a statistically steady state of such Langmuir eddies.

The RDT model therefore throws light upon the dynamics within the Stokes layer of the ocean mixed layer, where the Stokes drift operates and the production of TKE by Stokes production balances dissipation. Deeper into the mixed layer turbulent transport of TKE balances dissipation, which Polton and Belcher (2007) suggest is mediated by downwelling jets originating in the convergence zones within the Stokes layer. Although turbulent transport is a nonlinear process, and therefore not captured in the RDT model, the flux of TKE comes from the Stokes layer, which is well modelled by RDT, and so it may well be that RDT estimates can be used to parameterise this flux. 


\section{Acknowledgement}

We are grateful for the constructive comments of two anonymous referees. M. A. C. T. acknowledges the financial support of Fundação para a Ciência e Tecnologia (FCT) under Project AWARE/PTDC-ATM/65125/2006.

\section{Appendix A. Discussion of the vorticity dynamics}

We return now to the vorticity equations to help understand the large differences between turbulence distorted rapidly by shear and by Stokes drift, and consider further the relationship to the Craik-Leibovich model of Langmuir circulation. In the presence of shear and Stokes drift the vorticity equations become

$$
\begin{aligned}
\frac{D \omega_{x}}{D t} & =\omega_{z}\left(\alpha_{S}+\alpha\right)+\alpha \frac{\partial u}{\partial y}=\omega_{z} \alpha_{S}+\alpha \frac{\partial v}{\partial x}, \\
\frac{D \omega_{y}}{D t} & =\alpha \frac{\partial v}{\partial y}, \\
\frac{D \omega_{z}}{D t} & =\alpha \frac{\partial w}{\partial y}=\alpha\left(\omega_{x}+\frac{\partial v}{\partial z}\right) .
\end{aligned}
$$

In each case $D / D t=\partial / \partial t+\left(U+U_{S}\right) \partial / \partial x$. The first term on the right of (A-1) for the streamwise vorticity is the vortex stretching term by the mean Eulerian and Stokes drift shear $\omega_{z}\left(\alpha+\alpha_{S}\right)$. The last term on the right hand side arises through deformation of the vorticity in the mean shear flow by the turbulence. These two terms have been rewritten as a vorticity component and a gradient of the spanwise velocity, $v$. The Eulerian shear part in the first term is partially cancelled by the deformation of the mean vorticity by the turbulent velocity $(\alpha \partial u / \partial y)$, leaving only $\alpha \partial v / \partial x$. This cancellation 
is the key aspect determining differences between shear and wave-distorted turbulence (see also Fig. 15 of TB2002).

The vertical vorticity equation has a vortex stretching term resulting from interaction of the turbulent velocity with the mean vorticity $(\alpha \partial w / \partial y)$. This term is written as a sum of $\alpha \omega_{x}$ and $\alpha \partial v / \partial z$. The equation for $\omega_{y}$ also contains a term involving $v$, corresponding physically to stretching of the mean spanwise vorticity by the turbulence.

From energy arguments, it can be shown that the variance of $v$ is not directly affected by energy production terms, but only by the redistribution of the turbulence energy through the pressure. For that reason, all terms involving $v$ in (A-1)-(A-3) will not be considered in the following schematic argument (they are retained in the full RDT calculations).

Equations (A-1)-(A-3) then show in a simplified way how the coupling between the components of the vorticity is different in the three cases of distortion by mean shear, Stokes drift and both mean shear and Stokes drift.

When the deformation is by mean shear only, the essential process acting is the conversion of streamwise into vertical vorticity by the term $\alpha \omega_{x}$ in the $\omega_{z}$ budget. This causes the dominance of the $u$ and $v$ velocity fluctuations in shear turbulence. (Although this is not the whole story. If only $\omega_{z}$ increased, $u$ and $v$ should tend to have the same intensity in highly sheared turbulence, which is known not to be the case (e.g. Lee et al., 1990). In fact, other components of vorticity, generated by the processes associated with the $v$ terms, must play a role in producing the approximately one-dimensional structure that highly sheared turbulence has).

When deformation is by the Stokes drift only, the situation is considerably 
simpler: $\omega_{y}$ and $\omega_{z}$ do not vary much, but $\omega_{x}$ strongly increases due to tilting and stretching of $\omega_{z}$ by the Stokes drift shear, as pointed out by TB2002. This situation, which corresponds to the dominance of $v$ and $w$ velocity fluctuations of approximately similar intensity, is consistent with streamwise rolls, or Langmuir circulations.

Finally, when both shear and the Stokes drift are present, if the terms involving $v$ are again ignored, (A-1)-(A-3) give a coupled differential equation set for $\omega_{x}$ and $\omega_{z}$, from which separate equations for each of these quantities can be isolated. It then results that both $\omega_{x}$ and $\omega_{z}$ grow exponentially in time, with a growth rate proportional to $\left(\alpha \alpha_{S}\right)^{1 / 2}$. This growth rate is typical of Langmuir circulations in a neutrally stratified ocean, as shown by Leibovich (1977).

\section{References}

Batchelor, G. K., Proudman, I., 1954. The effect of rapid distortion of a fluid in turbulent motion. Quarterly Journal of Mechanics and Applied Mathematics 7, 83-103.

Brocchini, M., Peregrine, D. H., 2001. The dynamics of strong turbulence at free surfaces. Part 1. Description. Journal of Fluid Mechanics 449, 225-254.

Cox, S. M., Leibovich, S., 1993. Langmuir circulations in a surface layer bounded by a strong thermocline. Journal of Physical Oceanography 23, $1330-1345$.

Craik, A. D. D., 1977. The generation of Langmuir circulations by an instability mechanism. Journal of Fluid Mechanics 81, 209-223. 
Craik, A. D. D., Leibovich, S., 1976. A rational model for Langmuir circulations. Journal of Fluid Mechanics 73, 401-426.

D'Asaro, E., 2001. Turbulent vertical kinetic energy in the ocean mixed layer. Journal of Physical Oceanography 31, 3530-3537.

Dubrulle, B., Laval, J.-P., Nazarenko, S., Zaboronski, O., 2004. A model for rapid stochastic distortions of small-scale turbulence. Journal of Fluid Mechanics 520, 1-21.

Durbin, P. A., 1981. Distorted turbulence in axisymmetric flow. Quarterly Journal of Mechanics and Applied Mathematics 34, 489-500.

Faller, A. J., Auer, S. J., 1988. The roles of Langmuir circulations in the dispersion of surface tracers. Journal of Physical Oceanography 18, 11081123.

Faller, A. J., Cartwright, R. W., 1983. Laboratory studies of Langmuir circulations. Journal of Physical Oceanography 13, 329-340.

Fung, J. C. H., Hunt, J. C. R., Malik, N. A., Perkins, R. J., 1992. Kinematic simulation of homogeneous turbulence by unsteady random Fourier modes. Journal of Fluid Mechanics 236, 281-318.

Garrett, C., 1996. Processes in the surface mixed layer of the ocean. Dynamics of Atmospheres and Oceans 23, 19-34.

Gartshore, I. S., Durbin, P. A., Hunt, J. C. R., 1983. The production of turbulent stress in a shear flow by irrotational fluctuations. Journal of Fluid Mechanics 137, 307-329. 
Grant, A. L. M., Belcher, S. E., 2009. Characteristics of Langmuir turbulence in the ocean mixed layer. Journal of Physical Oceanography, in press.

Harcourt, R. R., D'Asaro, E. A., 2008. Large-eddy simulation of Langmuir turbulence in pure wind seas. Journal of Physical Oceanography 38, 15421562.

Hunt, J. C. R., 1973. A theory of flow round two-dimensional bluff bodies. Journal of Fluid Mechanics 61, 625-706.

Hunt, J. C. R., 1984. Turbulence structure in thermal convection and shearfree boundary layers. Journal of Fluid Mechanics 138, 161-184.

Hunt, J. C. R., Graham, J. M. R., 1978. Free stream turbulence near plane boundaries. Journal of Fluid Mechanics 84, 209-235.

Hunt, J. C. R., Kawai, H., Ramsey, S. R., Pedrizetti, G., Perkins, R. J., 1990. A review of velocity and pressure fluctuations in turbulent flows around bluff bodies. Journal of Wind Engineering and Industrial Aerodynamics $35,49-85$.

Kaneda, Y., Ishihara, T., Yokokawa, M., Itakura, K., Uno, A, 2003. Energy dissipation rate and energy spectrum in high resolution direct numerical simulations of turbulence in a periodic box. Physics of Fluids 15, L21-L24.

Kantha, L. H., Clayson, C. A., 2004. On the effect of surface gravity waves on mixing in the oceanic mixed layer. Ocean Modelling 6, 101-124.

Lee, M. J., Hunt, J. C. R., 1989. The structure of sheared turbulence near a plane boundary, in: Durst, F. et al. (Eds.), Seventh Symposium on 
Turbulent Shear Flows, Stanford University, Stanford, California, USA, Aug. 21-23 1989, pp. 8.1.1-8.1.6.

Lee, M. J., Kim, J., Moin, P., 1990. Structure of turbulence at high shear rate. Journal of Fluid Mechanics 216, 561-583.

Leibovich, S., 1977. On the evolution of the system of wind drift currents and Langmuir circulations in the ocean. Part 1. Theory and averaged current. Journal of Fluid Mechanics 79, 715-743.

Leibovich, S., 1980. On wave-current interaction theories of Langmuir circulations. Journal of Fluid Mechanics 99, 715-724.

Leibovich, S., 1983. The form and dynamics of Langmuir circulations. Annual Review of Fluid Mechanics 15, 391-427.

Leibovich, S., Paolucci, S., 1981. The instability of the ocean to Langmuir circulations. Journal of Fluid Mechanics 102, 141-167.

Leibovich, S., Radhakrishnan, K., 1977. On the evolution of the system of wind drift currents and Langmuir circulations in the ocean. Part 2: Structure of the Langmuir vortices. Journal of Fluid Mechanics 80, 481-507.

Li, M., Garrett, C., Skyllingstad, E., 2005. A regime diagram for classifying turbulent large eddies in the upper ocean. Deep-sea Research 52, 259-278.

Magnaudet, J., 2003. High-Reynolds number turbulence in a shear-free boundary layer: revisiting the Hunt-Graham theory. Journal of Fluid Mechanics 484, 167-196. 
Mann, J., 1994. The spatial structure of neutral atmospheric surface-layer turbulence. Journal of Fluid Mechanics 273, 141-168.

McWilliams, J. C., Sullivan, P. P., 2001. Vertical mixing by Langmuir circulations. Spill Science Technology 6, 225-237.

McWilliams, J. C., Sullivan, P. P., Moeng, C.-H., 1997. Langmuir turbulence in the ocean. Journal of Fluid Mechanics 334, 1-30.

Melville, W. K., Shear, R., Veron, F., 1998. Laboratory measurements of the generation and evolution of Langmuir circulations. Journal of Fluid Mechanics 364, 31-58.

Nepf, H. M., Monismith, S. G., 1991. Experimental study of wave-induced longitudinal vortices. Journal of Hydraulic Engineering 117, 1639-1649.

Nepf, H. M., Cowen, E. A., Kimmel, S. J., Monismith, S. J., 1995. Longitudinal vortices beneath breaking waves. Journal of Geophysical Research 100, 16211-16221.

Noh, Y. and Min, H. S., 2004. Large eddy simulation of the ocean mixed layer: the effects of wave breaking and Langmuir circulation. Journal of Physical Oceanography 34, 720-735.

Pearson, B. R., Krogstad, P.-A., van de Water, W., 2002. Measurements of the turbulent energy dissipation rate. Physics of Fluids 14, 1288-1290.

Perkins, R. J., Carruthers, D. J., Drayton, M. J., Hunt, J. C. R., 1990. Turbulence and diffusion at density interfaces, in: Hewitt, G. F., Mayinger, 
F., Riznic, J. R. (Eds.), Phase-interface phenomena in multiphase flow, Hemisphere, pp. 21-30.

Pluddemann, A. J., Smith, J. A., Farmer, D. M., Weller, R. A., Crawford, W. R., Pinkel, R., Vagle, S., Gnanadesikan, A., 1996. Structure and variability of Langmuir circulation during the Surface Waves Processes Program. Journal of Geophysical Research 101, 3525-3543.

Polton, J. A., Lewis, D. M., Belcher, S. E., 2005. The role of wave-induced Coriolis-Stokes forcing on the wind-driven mixed layer. Journal of Physical Oceanography 35, 444-457.

Polton, J. A., Belcher, S. E., 2007. Langmuir turbulence and deeply penetrating jets in an unstratified mixed layer. Journal of Geophysical Research 112, C09020.

Skyllingstad, E. D., 2001. Scales of Langmuir circulation generated using a large-eddy simulation model. Spill Science Technology 6, 239-246.

Skyllingstad, E. D., Dembo, D. W., 1995. An ocean large-eddy simulation of Langmuir circulations and convection in the surface mixed layer. Journal of Geophysical Research 100, 8501-8522.

Smith, J. A., 1998. Evolution of Langmuir circulation during a storm. Journal of Geophysical Research 103, 12649-12668.

Teixeira, M. A. C., Belcher, S. E., 2000. Dissipation of shear-free turbulence near boundaries. Journal of Fluid Mechanics 422, 167-191. 
Teixeira, M. A. C., Belcher, S. E., 2002. On the distortion of turbulence by a progressive surface wave. Journal of Fluid Mechanics 458, 229-267.

Teixeira, M. A. C., Belcher, S. E., 2006. On the initiation of surface waves by turbulent shear flow. Dynamics of Atmospheres and Oceans 41, 1-27.

Tennekes, H., Lumley, J. L., 1972. A first course in turbulence, the MIT Press, Cambridge, Massachusetts, and London, England.

Thorpe, S. A., 2001. Langmuir circulation and the dispersion of oil spills in shallow seas. Spill Science Technology 6, 213-223.

Thorpe, S. A., 2004. Langmuir circulation. Annual Review of Fluid Mechanics $36,55-77$.

Townsend, A. A., 1970. Entrainment and the structure of turbulent flow. Journal of Fluid Mechanics 41, 13-46.

Tsai, W.-T., Chen, S.-M., Moeng, C.-H., 2005. A numerical study on the evolution and structure of a stress-driven free-surface turbulent shear flow. Journal of Fluid Mechanics 545, 163-192.

Turfus, C., Hunt, J. C. R., 1987. A stochastic analysis of the displacements of fluid elements in inhomogeneous turbulence using Kraichnan's method of random modes, in Comte-Bellot, G., Mathieu, J. (Eds.), Advances in Turbulence. Springer-Verlag, Berlin, pp. 191-203. 

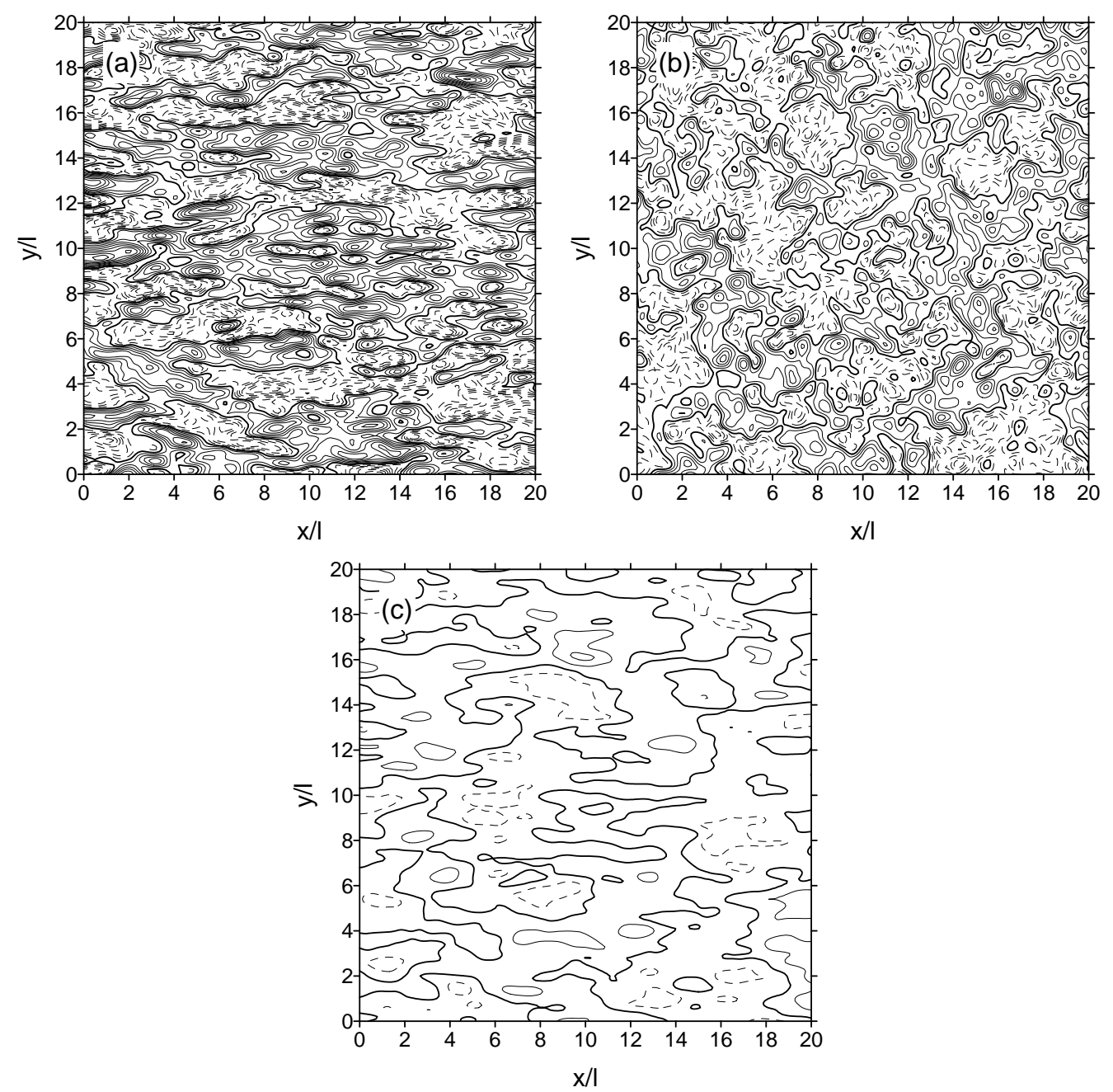

Figure 1: 

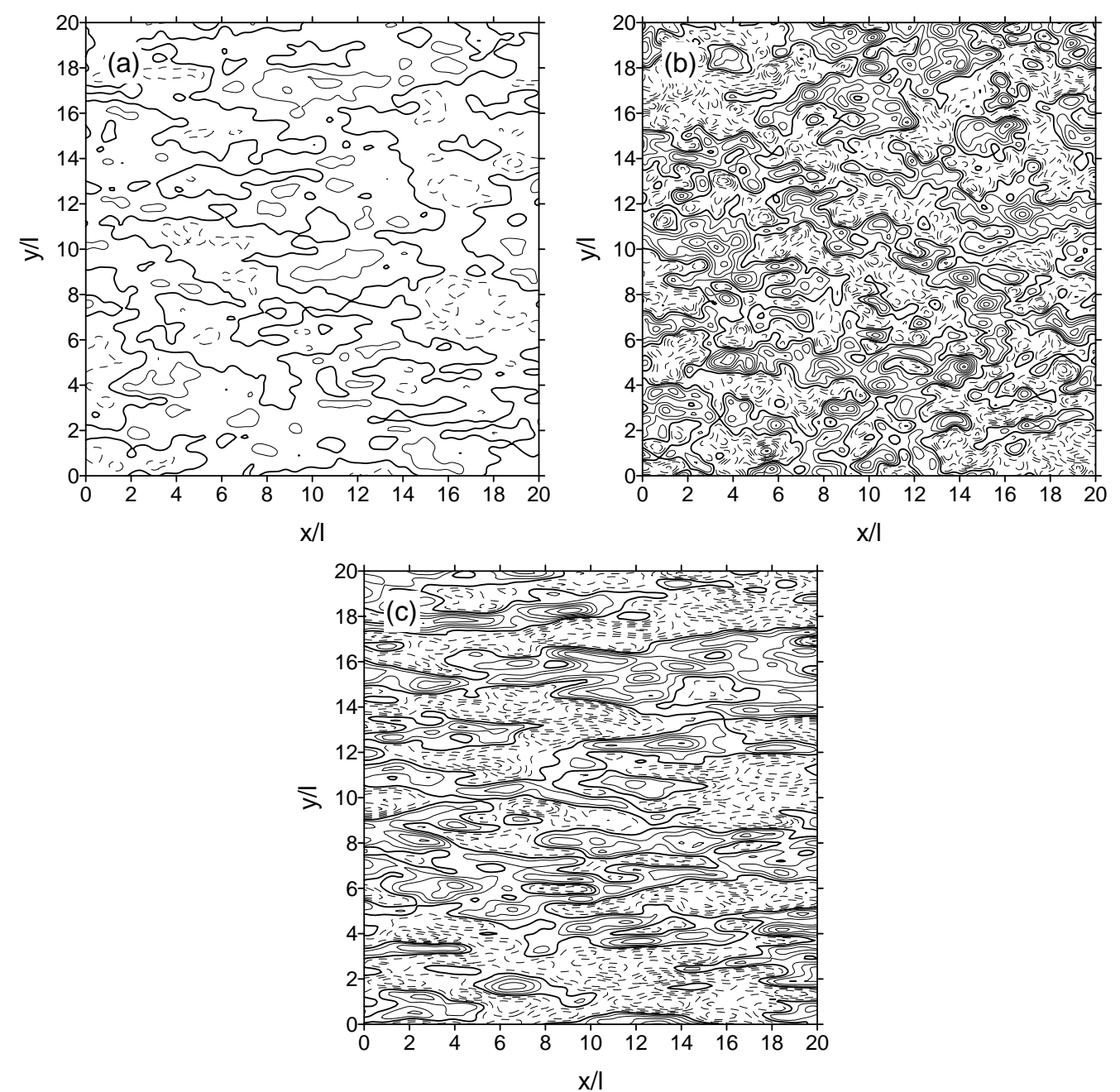

Figure 2: 


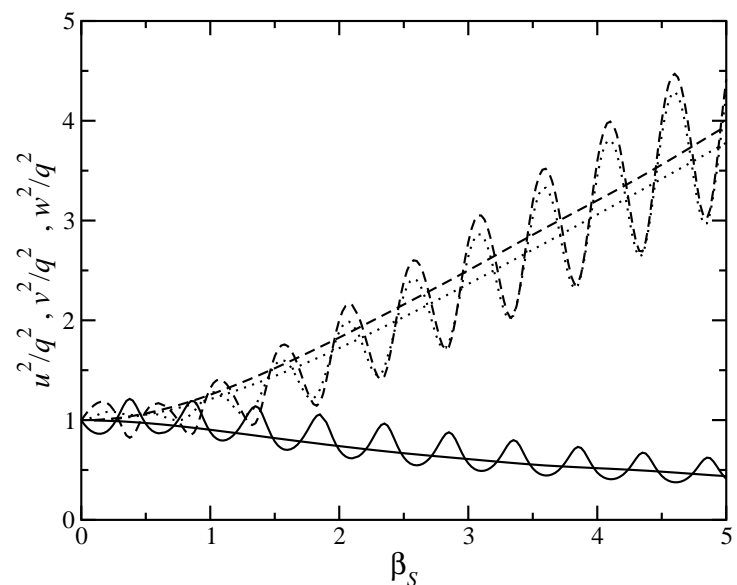

Figure 3: 

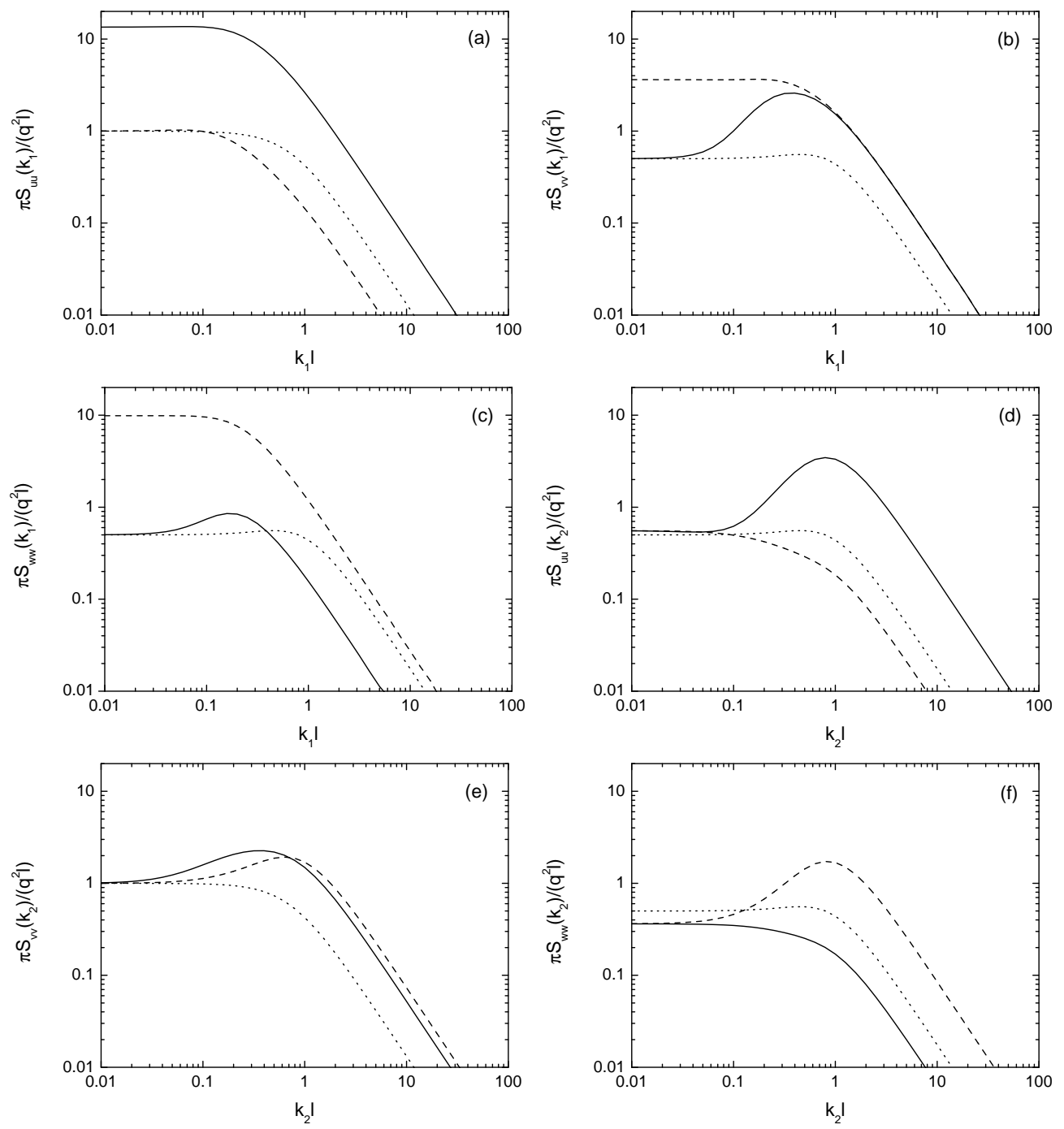

Figure 4: 

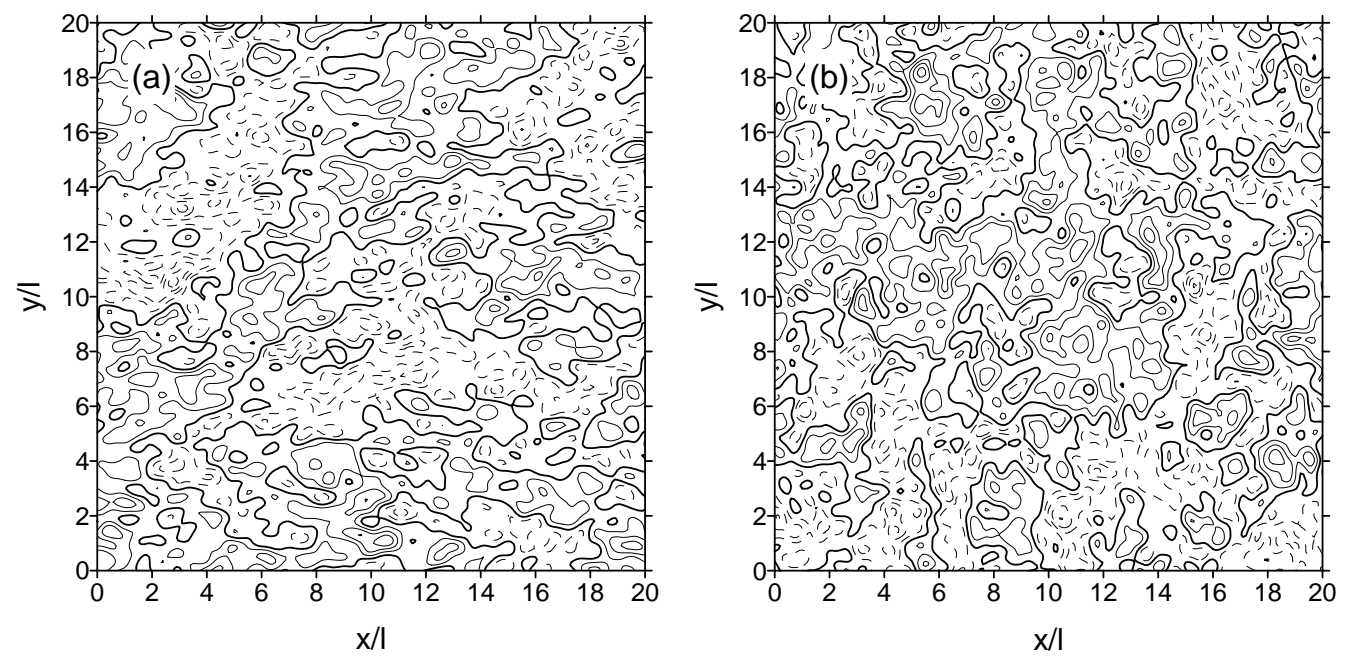

Figure 5: 

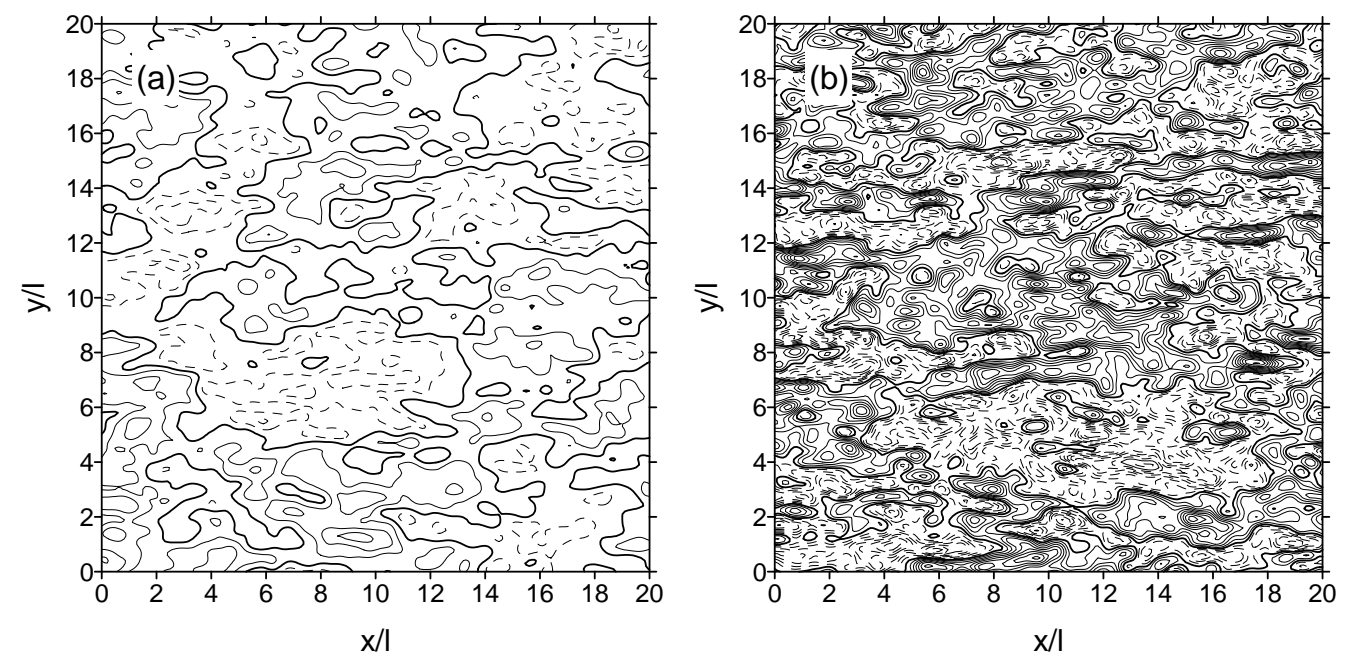

Figure 6: 

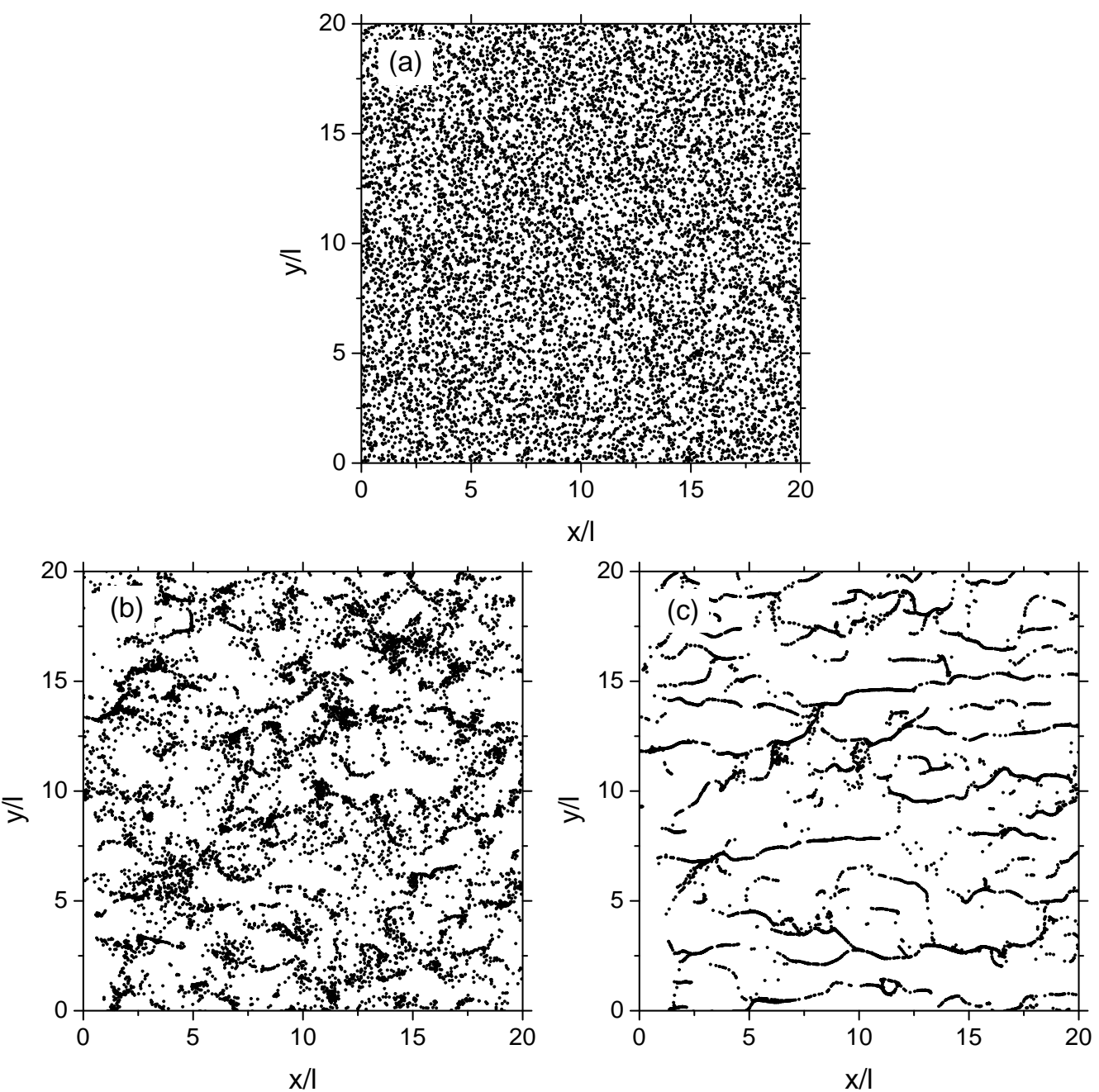

Figure 7: 


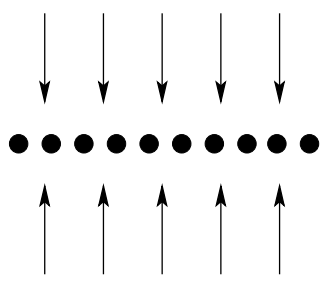

Wave

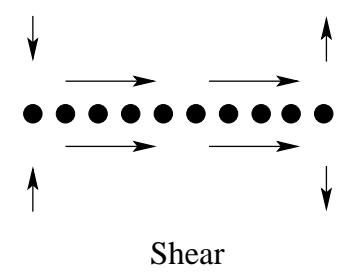

Shear

Figure 8: 

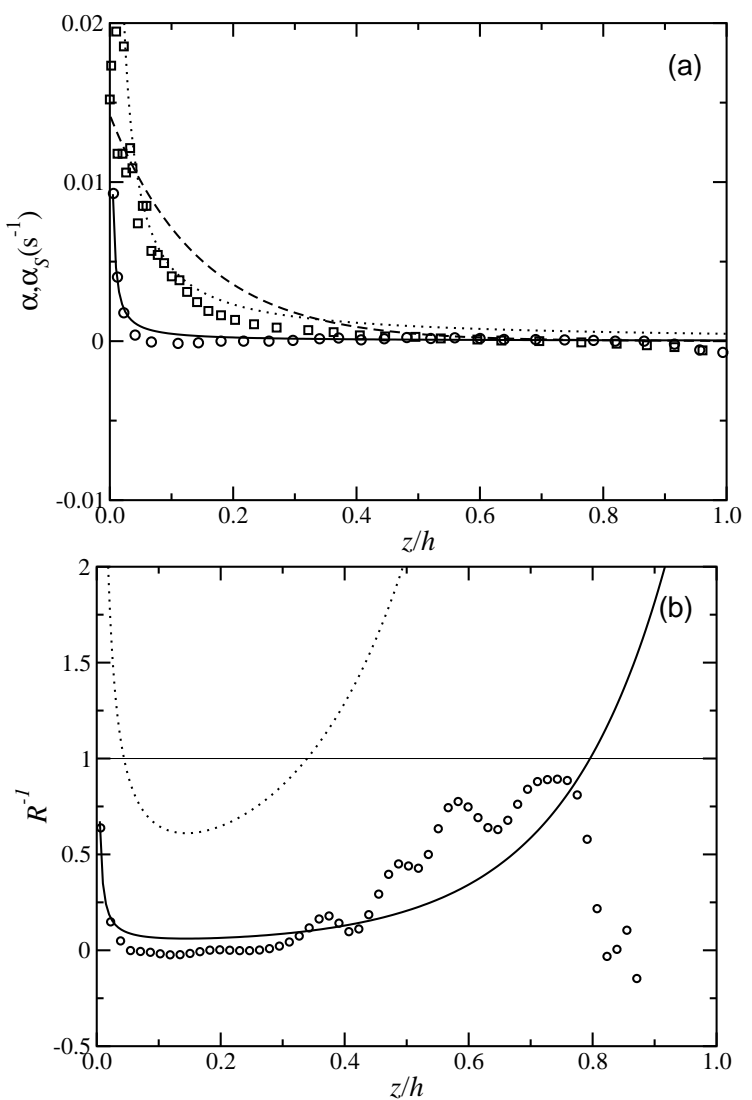

Figure 9: 

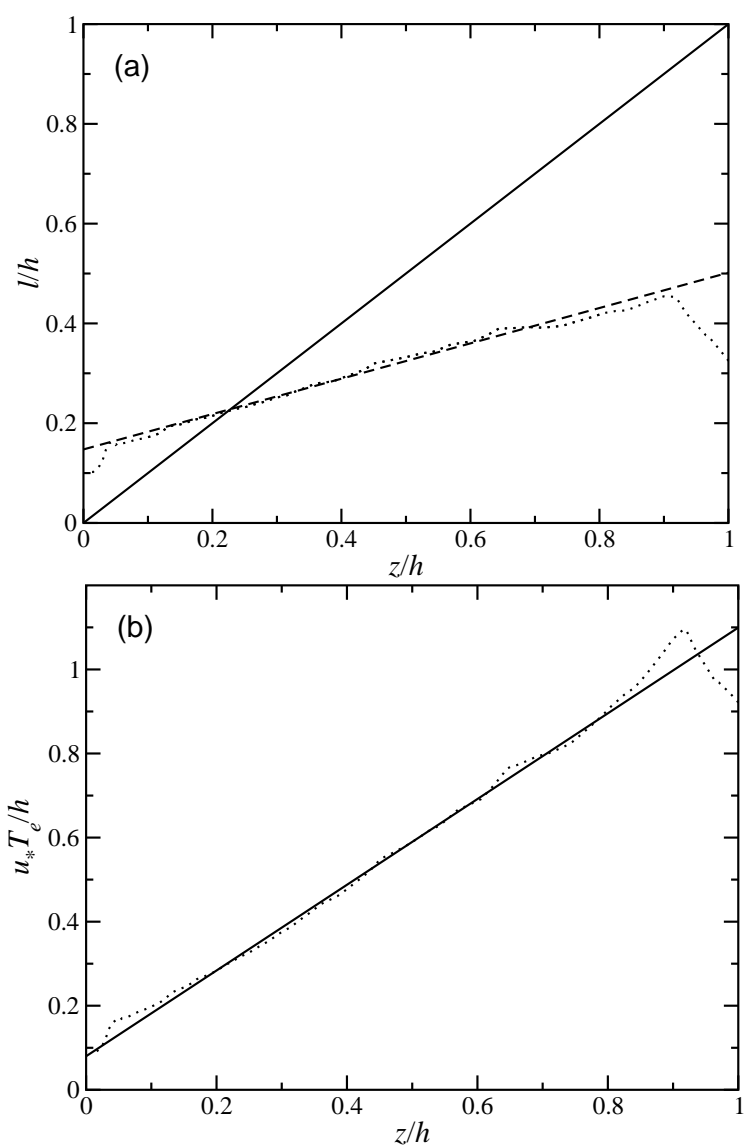

Figure 10: 


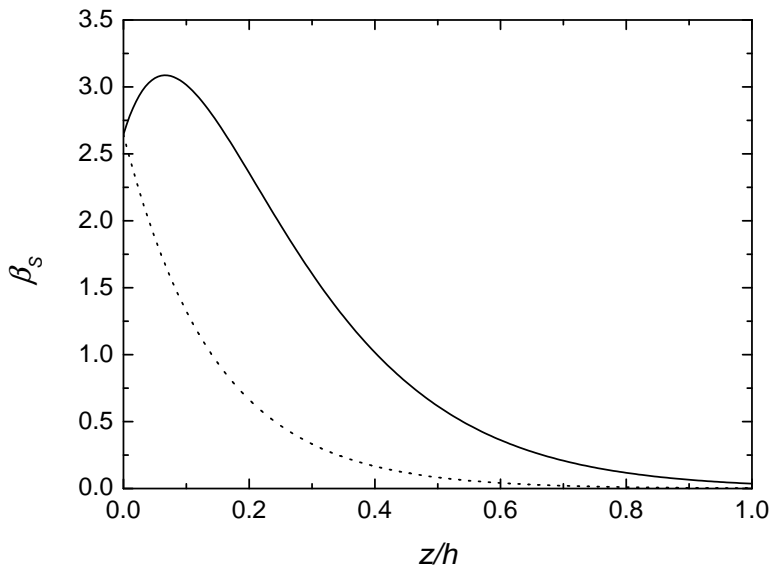

Figure 11: 

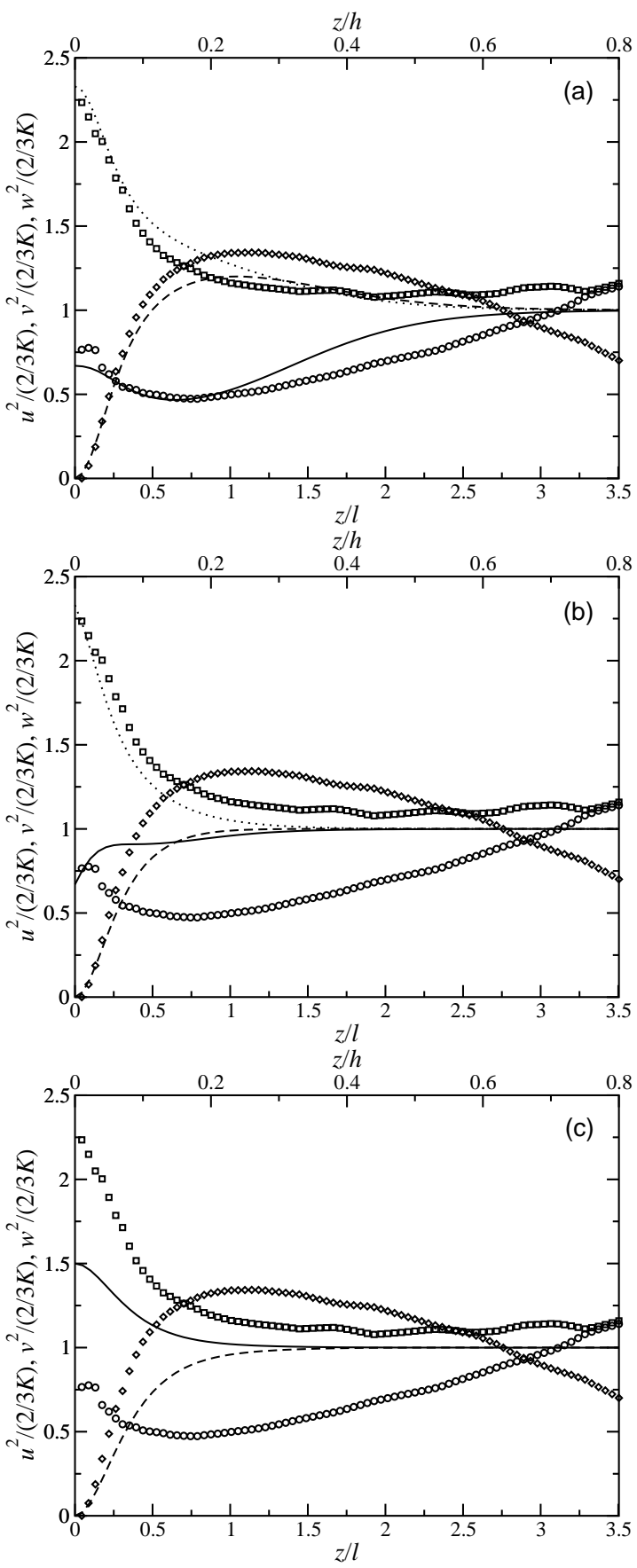

Figure 12: 

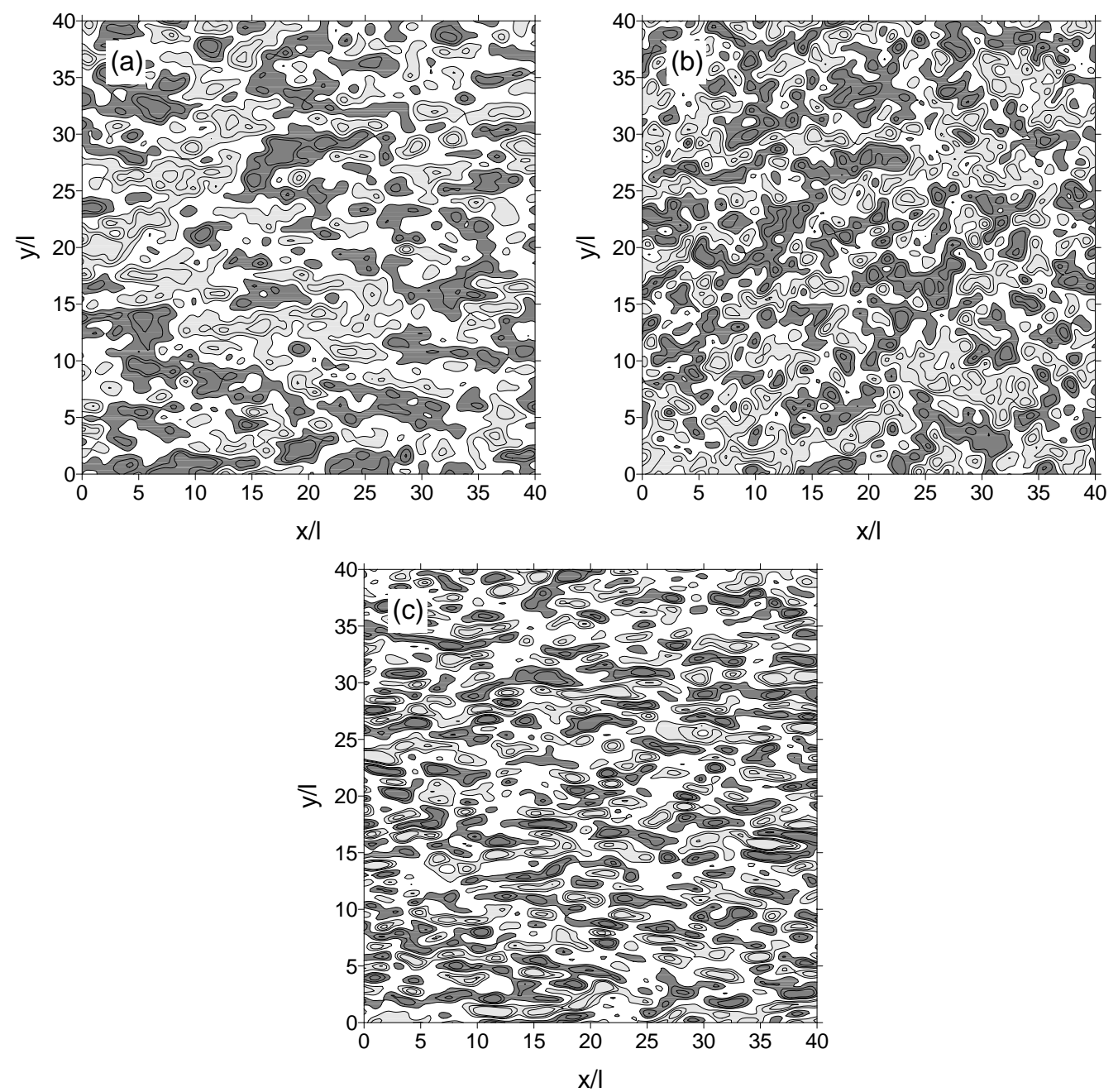

Figure 13: 


\section{Figure captions}

Figure 1: Normalised velocity field in turbulence distorted by the shear flow (11) at a depth $z / l=10$ for a dimensionless time $\beta=5$. Spatial distances normalised by $l$ and velocity fluctuations normalised by $q$. Contour spacing: 1. Solid lines: non-negative values, dashed lines: Negative values. (a) $u / q$, (b) $v / q$, (c) $w / q$.

Figure 2: As Fig. 1, but for turbulence distorted by a surface wave (12) for $\beta_{S}=5$.

Figure 3: Normalised velocity variances in turbulence distorted by a surface wave as a function of $\beta_{S}$. Wavy lines: using (12), smooth lines: using (14). Solid lines: $\overline{u^{2}} / q^{2}$, dotted lines: $\overline{v^{2}} / q^{2}$, dashed lines: $\overline{w^{2}} / q^{2}$.

Figure 4: Normalised spectra of the turbulent velocity fluctuations as a function of dimensionless wavenumber for $\beta=5$ or $\beta_{S}=5$. Solid lines: shear turbulence, dashed lines: wave-distorted turbulence, dotted lines: initial spectrum. (a),(d): Spectra of $u,(\mathrm{~b}),(\mathrm{e})$ : Spectra of $v,(\mathrm{c}),(\mathrm{f})$ : Spectra of $w$. (a)-(c): Along $x,(\mathrm{~d})-(\mathrm{f})$ : Along $y$.

Figure 5: Normalised velocity field in turbulence distorted by shear (11) at the surface for $\beta=5$. Spatial distances normalised by $l$ and velocity fluctuations normalised by $q$. Contour spacing: 1 . Solid lines: non-negative values, dashed lines: negative values. (a) $u / q$, (b) $v / q$.

Figure 6: As Fig. 5, but for turbulence distorted by a surface wave (12) for $\beta_{S}=5$. 
Figure 7: Tracer particles released at the surface and transported by the turbulence over an eddy turn-over time, $q t / l=1$. Distances normalised by l. (a) Initial positions, (b) Shear turbulence at $\beta=5$, (c) Wave-distorted turbulence at $\beta_{S}=5$.

Figure 8: Schematic diagram showing what processes lead to the alignment of surface tracers in wave-distorted turbulence and in shear turbulence. Arrows: direction of motion, circles: tracer particles.

Figure 9: (a) Profile of the shear rate in experiment $S / \infty$ (squares) and experiment $E / 0.3$ of MSM97 (circles), for a logarithmic profile with $u_{*}=$ $6.1 \times 10^{-3} \mathrm{~ms}^{-1}$ (dotted line) and for a logarithmic profile with $u_{*}=0.61 \times$ $10^{-3} \mathrm{~ms}^{-1}$ (solid line), and of the strain rate associated with the Stokes drift of the wave (dashed line). (b) Profile of $R^{-1}$ in experiment $E / 0.3$ of MSM97 (circles) and from (17) for $u_{* s}=0.61 \times 10^{-3} \mathrm{~ms}^{-1}$ (solid line) and $u_{* s}=$ $6.1 \times 10^{-3} \mathrm{~ms}^{-1}$ (dotted line).

Figure 10: Profiles of the normalised integral length scale and eddy turn-over time derived from the data of MSM97. (a) Integral length scale. Dotted line: LES data, dashed line: linear fit between $z / h=0.1$ and $z / h=0.8$, solid line: $z / l=1$. (b) Eddy turn-over time. Dotted line: LES data, solid line: linear fit between $z / h=0.1$ and $z / h=0.8$.

Figure 11: Dimensionless time $\beta_{S}$ used in the RDT and KST models as a function of depth from (27) using the data from experiment E0.3 of MSM97 (solid line) and using the surface eddy turn-over time everywhere (dotted line). 
Figure 12: Profiles of the normalised turbulent velocity variances. Symbols: data from MSM97, lines: RDT of turbulence distorted by a surface wave. Solid lines and circles: $\overline{u^{2}} /(2 / 3 K)$, dotted lines and squares: $\overline{v^{2}} /(2 / 3 K)$, dashed lines and diamonds: $\overline{w^{2}} /(2 / 3 K)$. (a) Depth-dependent eddy turnover time, (b) Constant eddy turn-over time, (c) Only with blocking effect (note that the solid and dotted lines are superimposed).

Figure 13: Velocity fluctuations in turbulence distorted by a surface wave at $z / l=0.40$ from KST for the conditions of experiment E/0.3 of MSM97. Dark shading: positive values, light shading: negative values. Contour spacings are equivalent to those used in MSM97: $u-( \pm 0.41, \pm 1.03, \pm 1.55)$, $v-( \pm 0.62, \pm 1.55, \pm 2.59), w-( \pm 0.52, \pm 1.03, \pm 1.55)$ (see text for details). (a) $u / q$ field, (b) $v / q$ field, (c) $w / q$ field. 